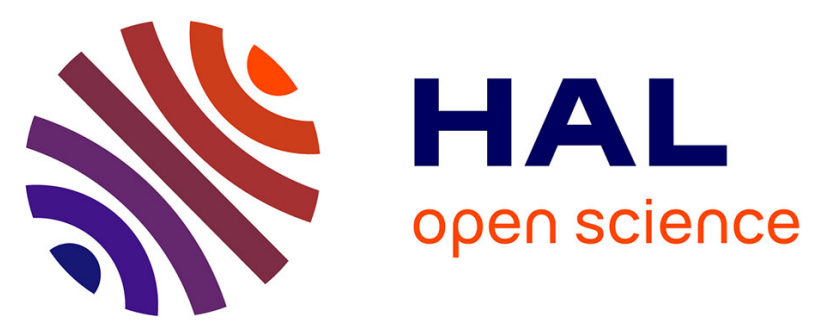

\title{
A strategy to reduce the sensitivity of inhomogeneous Magnetization Transfer (ihMT) imaging to radiofrequency transmit field variations at $3 \mathrm{~T}$
}

\author{
Lucas Soustelle, Thomas Troalen, Andreea Hertanu, Samira Mchinda,
} Jean-philippe Ranjeva, Maxime Guye, Gopal Varma, David Alsop, Guillaume Duhamel, Olivier Girard

\section{To cite this version:}

Lucas Soustelle, Thomas Troalen, Andreea Hertanu, Samira Mchinda, Jean-philippe Ranjeva, et al.. A strategy to reduce the sensitivity of inhomogeneous Magnetization Transfer (ihMT) imaging to radiofrequency transmit field variations at $3 \mathrm{~T}$. Magnetic Resonance in Medicine, 2021, 10.1002/mrm.29055 . hal-03429467

\section{HAL Id: hal-03429467 https://hal.science/hal-03429467}

Submitted on 15 Nov 2021

HAL is a multi-disciplinary open access archive for the deposit and dissemination of scientific research documents, whether they are published or not. The documents may come from teaching and research institutions in France or abroad, or from public or private research centers.
L'archive ouverte pluridisciplinaire $\mathbf{H A L}$, est destinée au dépôt et à la diffusion de documents scientifiques de niveau recherche, publiés ou non, émanant des établissements d'enseignement et de recherche français ou étrangers, des laboratoires publics ou privés. 


\title{
A strategy to reduce the sensitivity of inhomogeneous Magnetization Transfer (ihMT) imaging to radiofrequency transmit field variations at $3 \mathrm{~T}$
}

\author{
Lucas Soustelle ${ }^{1,2}$, Thomas Troalen ${ }^{3}$, Andreea Hertanu ${ }^{1,2}$, Samira Mchinda ${ }^{1,2}$, Jean-Philippe \\ Ranjeva $^{1,2}$, Maxime Guye ${ }^{1,2}$, Gopal Varma ${ }^{4}$, David C. Alsop ${ }^{4}$, Guillaume Duhamel ${ }^{1,2}$, \\ Olivier M. Girard ${ }^{1,2^{*}}$ \\ 1. Aix Marseille Univ, CNRS, CRMBM, Marseille, France \\ 2. APHM, Hôpital Universitaire Timone, CEMEREM, Marseille, France \\ 3. Siemens Healthcare SAS, Saint-Denis, France \\ 4. Division of MR Research, Radiology, Beth Israel Deaconess Medical Center, Harvard Medical \\ School, Boston, MA, United States
}

\begin{abstract}
*Corresponding author: Olivier M. Girard, PhD (olivier.girard@univ-amu.fr), Aix Marseille Université, Centre de Résonance Magnétique Biologique et Médicale, CRMBM UMR 7339 - Faculté de Médecine, 27 Boulevard Jean Moulin, 13005 Marseille, France
\end{abstract}

Word count: 5426/5000 


\section{Abstract (246/250 words)}

Purpose: To minimize the sensitivity of inhomogeneous Magnetization Transfer gradient-echo (ihMTGRE) imaging to RF transmit field $\left(\mathrm{B}_{1}^{+}\right)$inhomogeneities at $3 \mathrm{~T}$.

Methods: The ihMT-GRE sequence was optimized by varying the concentration of the RF saturation energy over time, obtained by increasing the saturation pulse power while extending the sequence repetition time (TR). Different protocols were tested using numerical simulations and human in vivo experiments in the brain white matter (WM) of healthy subjects at $3 \mathrm{~T}$. The sensitivity of the inMT ratio (ihMTR) to $\mathrm{B}_{1}{ }^{+}$variations was investigated by comparing measurements obtained at nominal transmitter adjustments and following a $20-\%$ global $B_{1}{ }^{+}$drop. The resulting relative variations $\left(\delta_{\text {ihMTR }}\right)$ were evaluated voxelwise as a function of the local $\mathrm{B}_{1}{ }^{+}$distribution. The reproducibility of the protocol providing minimal $\mathrm{B}_{1}{ }^{+}$bias was assessed in a test-retest experiment.

Results: In line with simulations, ihMT-GRE experiments conducted at high concentration of the RF energy over time demonstrated strong reduction of the $\mathrm{B}_{1}{ }^{+}$inhomogeneity effects in the human WM. Under the optimal conditions of $350-\mathrm{ms}$ TR and $3-\mu \mathrm{T}$ RMS saturation power, $73 \%$ of all WM voxels presented $\delta_{\text {ihMTR }}$ below $10 \%$. Reproducibility analysis yielded a close-to-zero systematic bias $(\Delta$ ihMTR $=-0.081 \%)$ and a high correlation $\left(\rho^{2}=0.977\right)$ between test and retest experiments.

Conclusion: Concentrating RF saturation energy in ihMT-GRE sequences mitigates the sensitivity of the inMTR to $\mathrm{B}_{1}{ }^{+}$variations and allows for clinical-ready ihMT imaging at $3 \mathrm{~T}$. This feature is of particular interest for high and ultra-high field applications.

Running title: Reducing the sensitivity of ihMT to $\mathrm{B}_{1}{ }^{+}$inhomogeneities

Keywords: ihMT, inhomogeneous magnetization transfer, radiofrequency field inhomogeneities, magnetization transfer, myelin, neuroimaging 


\section{Introduction}

Inhomogeneous Magnetization Transfer (ihMT) imaging is a recent MRI technique (1) that has demonstrated good sensitivity and specificity to myelination (2). This technique, extended from conventional magnetization transfer (MT), allows isolating the dipolar order contribution to the MT effects generated in semi-solid tissue components (3). Off-resonance irradiation induces significant dipolar order primarily in molecules where the dipolar order relaxation time, $T_{1 D}$, is long, as in myelinated white matter (WM) (4). IhMT is typically acquired by obtaining MT images with radiofrequency (RF) irradiation preparation both at a single frequency offset and at two symmetric frequency offsets. The ihMT image is generated by a linear combination of single- and dual-offset MT images. The normalization by a reference image acquired without off-resonance saturation allows deriving a semi-quantitative ihMT ratio (ihMTR) image. To date, ihMT has been used in vivo in both rodent and human in the context of myelin imaging applications and has shown great potential for the characterization of both healthy and pathological WM (2,5-11).

When performed on scanners at relatively low static magnetic field (e.g., $1.5 \mathrm{~T}$ ) or in small fields of view such as in small animal imaging applications, a rather homogeneous RF transmit field $\left(\mathrm{B}_{1}^{+}\right)$is typically observed using volume emitter coils. However, since ihMT is an RF saturation-based MRI method with a high intrinsic sensitivity to $\mathrm{B}_{1}{ }^{+}$values, special attention must be paid to the spatial variations of $\mathrm{B}_{1}{ }^{+}$when applied at high field or/and when large samples are targeted, as spurious variations of the ihMTR may occur.

In a previous study, Mchinda et al. have reported different $\mathrm{B}_{1}{ }^{+}$dependence regimes of ihMTR as a function of the RF saturation characteristics using an ihMT gradient-echo (ihMT-GRE) sequence at $1.5 \mathrm{~T}$ in the human brain (12). More specifically it was shown that concentration of RF saturation energy over time (i.e., reduction of the RF saturation duty-cycle at constant averaged power over the sequence repetition time) yielded low variations of ihMTR with respect to the RMS saturation power above a certain value, hence holding promise for high field applications. Low RF duty-cycle conditions also provided remarkable enhancement of the ihMT sensitivity $(2,12,13)$, making them strongly desirable for optimized inMT imaging.

In this work, we expand upon these results and present a thorough analysis of the sensitivity of ihMTR to $\mathrm{B}_{1}{ }^{+}$variations. The analyses combined simulations and in vivo experiments to evaluate the response of ihMTR to a non-uniform $\mathrm{B}_{1}{ }^{+}$spatial distribution as well as to a $20-\%$ global $\mathrm{B}_{1}{ }^{+}$drop. Results were used to guide the optimization of low RF duty-cycle ihMT-GRE sequence at $3 \mathrm{~T}$ for human brain 
applications. In vivo reproducibility was also investigated using a selected set of parameters providing minimal sensitivity to $\mathrm{B}_{1}{ }^{+}$inhomogeneities.

\section{Methods}

Experiments were performed on a 3T clinical scanner (Vida, software version XA20A, Siemens Healthineers, Erlangen, Germany) with body coil transmission and a 32-channel receive head coil on

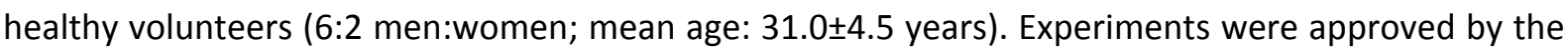
institutional ethics committee on clinical investigations (CRMBM, Marseille), and written informed consent was obtained from each participant before the study.

\section{IhMT sequence}

A non-selective sagittal 3D ihMT-GRE sequence analogous to the one described in Ref. (12) was used in this study (Figure 1) and is available upon request. The sequence, which interleaves MT preparation modules with multiple gradient- and RF-spoiled GRE readout modules ( $\mathrm{N}_{\text {GRE }}$ GRE kernels of individual duration $\mathrm{TR}_{\text {sub }}$ ) was run for 4 different MT preparation modules, allowing the acquisition of single offset $\mathrm{MT}^{+}$and $\mathrm{MT}^{-}$images and dual-offset $\mathrm{MT}^{+-}$and $\mathrm{MT}^{-+}$images, and an additional unsaturated reference scan. The reference image acquired with zero MT power will be referred to as $\mathrm{MT}_{0}$ in the following, to differentiate this slightly $T_{1}$-weighted image from $M_{0}$, the equilibrium magnetization. $\mathrm{MT}^{+}$and $\mathrm{MT}^{-}$ images were obtained using bursts of RF saturation pulses (individual duration PW and interpulse repetition time $\Delta \mathrm{t})$ at positive $(+\Delta \mathrm{f})$ and negative $(-\Delta \mathrm{f})$ offset frequency, respectively. For the dualoffset $\mathrm{MT}^{+-}$and $\mathrm{MT}^{-+}$images, the offset frequency was alternated from $+\Delta f$ to $-\Delta f$ for each successive pulse, providing a $T_{1 D}$ contrast weighted towards the long $T_{1 D}$ of myelinated tissues (14). All RF saturation pulses were followed by a gradient spoiler. The $\mathrm{MT}_{0}$ image was obtained by switching off the off-resonance pulse amplitude while maintaining the gradient spoilers. To mitigate the specific absorption rate (SAR) over the whole acquisition, a 50-\% partial Fourier saturation approach was used with $\mathrm{k}$-space lines acquired in a centric-out fashion in the $\mathrm{k}_{\mathrm{y}}-\mathrm{k}_{\mathrm{z}}$ plane and following a Cartesian spiral trajectory as previously described by Mchinda et al. (12). IhMTR images were computed as:

$$
\left.\operatorname{ihMTR}=\frac{\mathrm{ihMT}}{\mathrm{MT}_{0}}, \text { with } \operatorname{ihMT}=\left(\mathrm{MT}^{+}+\mathrm{MT}^{-}\right)-\left(\mathrm{MT}^{+-}+\mathrm{MT}^{-+}\right) \quad \text { (eq. } 1\right)
$$

The time-averaged saturation RF power was characterized by the root mean square $B_{1}$ calculated over the repetition time of the sequence $\left(\mathrm{B}_{1, \mathrm{RMS}}{ }^{\mathrm{TR}}\right)$ as:

$$
B_{1, \mathrm{RMS}}^{\mathrm{TR}}=B_{1, \mathrm{RMS}}^{\mathrm{pulse}} \times \sqrt{\frac{N_{p} \times P W}{\mathrm{TR}}} \quad(\text { eq. } 2)
$$


where $N_{p}$ is the number of consecutive RF pulses applied within a saturation burst every TR, PW is the pulse duration, and TR is the ihMT-GRE sequence repetition time. $B_{1, R M s}{ }^{\text {pulse }}$ is the root mean square $B_{1}$ calculated over a single saturation pulse, given by:

$$
\left.B_{1, \mathrm{RMS}}^{\text {pulse }}=\sqrt{\mathrm{p}_{2}} \times B_{1, \text { peak }}, \text { with } \mathrm{p}_{2}=\frac{1}{\tau} \int_{0}^{\tau} A(t)^{2} d t \text { (eq. } 3\right)
$$

where $B_{1, \text { peak }}$ is the peak amplitude of the pulse, and $p_{2}$ the normalized power integra I calculated from its normalized shape $A(t)$ defined over the duration $\tau$.

For a fixed preparation, varying the sequence repetition time value while keeping all other parameters constant leads to variable $B_{1, R M S} S^{T R}$ (eq. 2). Alternatively, constant $B_{1, R M S}{ }^{T R}$ is achieved for different TRs by adjusting the $B_{1, \text { peak }}$ value of the saturation pulses (eq. 3), as illustrated in Figure 1 . For short TR (Figure 1a), the deposition of saturation energy is rather distributed within TR (high RF duty-cycle conditions), while increasing TR at constant $B_{1, R M S}{ }^{T R}$ leads to the concentration of the energy deposition within TR (Figure 1b; low RF duty-cycle conditions). The transition from high to low duty-cycle at constant $B_{1, R M S}{ }^{T R}$ implies increasing $B_{1, \text { peak }}$ value, and thus the instantaneous saturation efficiency per pulse.

The value of $B_{1, \text { peak }}$ is limited by hardware constraints, especially in clinical MRI systems. Hence, increasing $B_{1, R M S}{ }^{T R}$ at maximum $B_{1, \text { peak }}$ and constant duty-cycle can be achieved by means of pulse shapes providing higher $p_{2}$ values (eq. 3). Tukey-shaped pulses (15), which consist of a family of cosinetapered windows with a variable parameter controlling the cosine fraction $(r)$, provide a suitable degree of freedom to modulate the power efficiency $\left(p_{2}=1-5 r / 8\right)$, allowing intermediate shapes from a rectangular one $\left(r=0 ; p_{2}=1.0\right)$ to a Hann one $\left(r=1 ; p_{2}=0.375\right)$. In the present study, Tukey-shaped pulses with $r=0.3$ were used. While the diminution of $r$ is beneficial to increase the power integral of Tukey-shaped pulses, it also modifies their spectral profile (both full width at half maximum and relative intensity of the sidelobes), potentially resulting in saturation of the free water pool due to nonzero power on-resonance, also known as direct saturation effects. This is particularly important in the context of the investigated frequency-alternated ihMT-GRE sequence since short off-resonance pulses (on the order of $1 \mathrm{~ms}$ ) are employed to generate dipolar order effects. A tradeoff is therefore to be made between the pulse duration, the cosine fraction and the associated power integral in order to maintain a desirable high $\mathrm{B}_{1, \mathrm{RMS}}{ }^{\mathrm{TR}}$ within acceptable direct saturation effects. Here, 1-ms Tukeyshaped $(r=0.3)$ pulses each followed by spoiling gradients were used for all experiments. Note that previous preliminary work $(16,17)$ used 0.5 -ms Tukey-shaped $(r=0.3)$ pulses without post-pulse spoiling gradients. However, such implementation resulted in significant direct saturation effects in 
poor $\mathrm{B}_{0}$-shimming conditions. Although the complete description of these effects is outside the scope of the present paper, more information is provided in the Discussion and Supporting Information.

\section{IhMT simulations}

The biophysical model used for simulating ihMTR values in WM considered two macromolecular pools exchanging with a liquid pool. Each macromolecular pool is associated with a distinct nonzero $T_{1 D}$ dipolar order reservoir bound to a Zeeman reservoir, as proposed by Carvalho et al. (14). In brief, BlochProvotorov equations accounting for magnetization transfer between the liquid and semi-solid pools were used to describe the dynamics of the magnetization of both Zeeman and dipolar order reservoirs. A matrix formulation of the system of differential equations was used as described in Ref. (14). The solution was calculated by piece-wise integration assuming time-constant events to describe the different steps of the interleaved ihMT-GRE sequence (saturation pulses, relaxation and exchanges, as well as readout pulses). The simulation framework is similar to the one previoulsy described in detail in Ref. (14). The saturation pulses were assumed of constant intensity with RMS power matched to that of the actual Tukey-shaped pulses. Readout pulses were considered instantaneous with a constant flip angle ( $F A)$, attenuating the free water longitudinal magnetization $\left(\mathrm{M}_{2, f}\right)$ by $\cos (\mathrm{FA})$, and their saturation effects on the bound pools were neglected (i.e., no modulation of the longitudinal magnetizations of the bound pools $M_{z, b, 1}$ and $\left.M_{z, b, 2}\right)$. A perfect spoiling of the free water transverse magnetization was assumed throughout the sequence. The simulation provide the steady-state magnetization of all pools obtained right after the inMT pulse burst (see Figure 2), that is just before the first readout pulse of the GRE train. A MATLAB (The Mathworks Inc., Natick, MA) implementation of this framework comprising codes used in the following simulations is made available at: [to be submitted on the Software Heritage platform (https://www.softwareheritage.org)]; the codes are provided as Supplementary Material.

Simulations of ihMTR expected in typical WM tissue using the ihMT-GRE sequence were performed with sequence parameters matched to the experimental protocols: $N_{p}=8$ Tukey-shaped off-resonance saturation pulses with $r=0.3, \mathrm{PW}=1.0 \mathrm{~ms}, \Delta \mathrm{t}=1.5 \mathrm{~ms}$ and $\Delta \mathrm{f}=7 \mathrm{kHz}$. The readout segment duration was fixed to $T R_{\text {sub }}=6.5 \mathrm{~ms}$, and the number of readouts was increased as TR was extended to provide a time efficient data sampling strategy as described in Ref. (12). Finally, the WM tissue parameters (subscripts $f$ and $b$ for free and bound pools, respectively) were set to values reported in the literature $(14,18): R_{1, f} / R_{1, b}=1.0 / 1.0 \mathrm{~s}^{-1}, T_{2, f}=22.0$ ms (i.e., $\left.R_{1, f} T_{2, f}=0.022\right), T_{2, b}=10.0 \mu s$, exchange rate $R=19.0 \mathrm{~s}^{-1}$, $M_{0, f} / M_{0, b}=1.0 / 0.16, T_{1 D, 1} / T_{1 D, 2}=10.0 / 0.4 \mathrm{~ms}$ and $f_{D}=0.5$. $f_{D}$ denotes the fractions of macromolecules associated with the long $T_{1 D}$ value $\left(T_{1 D, 1}\right)$, and $\left(1-f_{D}\right)$ the fraction associated with the short component $\left(T_{1 D, 2}\right)$, hence splitting the thermal equilibrium magnetization of the macromolecular pool $\left(M_{0, b}\right)$ into 
$M_{0, b, 1}=f_{D} M_{0, b}$ and $M_{0, b, 2}=\left(1-f_{D}\right) M_{0, b}$. Both macromolecular pools are described with identical $R_{1, b}, T_{2, b}$ and $\mathrm{R}$ values. Note that these biophysical parameters are not intended to accurately correspond to detailed WM tissue characteristics, but rather represent a reasonable choice to yield realistic ihMTR signal predictions along the explored model dimensions.

IhMTR was simulated for three different energy concentration schemes (TR of 38, 194 and 350 ms, corresponding to $\mathrm{N}_{\text {GRE }}$ of 4,28 and 52 , respectively) as a function of $B_{1, R M S}{ }^{T R}$ spanning from $0.25 \mu T$ to $5.0 \mu \mathrm{T}$ at a fixed readout $\mathrm{FA}$ of $5^{\circ}$; and as a function of FA spanning from 1 to $20^{\circ}$ at fixed $\mathrm{B}_{1, \mathrm{RMS}} \mathrm{T}^{\mathrm{TR}}=3 \mu \mathrm{T}$. In addition, ihMTR was simulated as a function of the energy concentration (TR spanning from 18.5 to $402 \mathrm{~ms}$ in steps of $\mathrm{TR}_{\text {sub }}=6.5 \mathrm{~ms}$ ) for nominal $B_{1, \mathrm{RMS}} \mathrm{TR}^{\mathrm{R}}=3 \mu \mathrm{T}$ and $\mathrm{FA}=5^{\circ}$. The response of ihMTR to $\mathrm{B}_{1}{ }^{+}$ was investigated by performing the simulations for nominal $\mathrm{B}_{1}{ }^{+}$value and nominal $\mathrm{B}_{1}{ }^{+} \pm 10 \%$ (i.e., $\mathrm{rB}_{1}{ }^{+}=0.9,1.0$ and 1.1; $\mathrm{rB}_{1}{ }^{+}$being defined as the ratio of actual to nominal $\mathrm{B}_{1}{ }^{+}$transmit field). Note that the investigated $\mathrm{rB}_{1}{ }^{+}$range corresponds to $\mathrm{B}_{1}{ }^{+}$inhomogeneities experimentally found in the human brain WM at $3 \mathrm{~T}$ (see Figure 4).

\section{MR imaging experiments}

Two studies were performed:

- Study 1: Fixed $B_{1, R M s}{ }^{T R}$, variable TRs

IhMT-GRE images were acquired in 3 control subjects with nominal $B_{1, R M S}{ }^{T R}$ of $3 \mu T$, and variable TRs (and hence variable $\mathrm{N}_{\text {GRE }}$ readouts; Table 1). All experiments were repeated with nominal $\mathrm{B}_{1}{ }^{+}$ deliberately attenuated by $20 \%$. This was achieved 1 ) with a regular, automatic, $\mathrm{B}_{1}{ }^{+}$adjustment corresponding to the nominal reference transmitter voltage $\left(\mathrm{V}_{\text {ref,100\% }}\right)$, and 2$)$ with the transmitter reference voltage manually reduced by $20 \%\left(\mathrm{~V}_{\text {ref,80\% }}\right)$.

\section{- Study 2: Reproducibility}

Based on the results of Study 1, the ihMT-GRE sequence configuration with strong energy concentration ( $B_{1, R M S}{ }^{T R}$ of $3 \mu T$, TR of $350 \mathrm{~ms}$ ) was selected for reproducibility assessment at 2-mm isotropic resolution in a test-retest protocol on two volunteers. Subjects were scanned once, moved out from the scanner for a 30-minute break and scanned a second time.

Common saturation parameters were: bursts of $N_{p}=8$ Tukey-shaped saturation pulses $(r=0.3)$ with a gradient spoiler after each off-resonance pulse, $\mathrm{PW} / \Delta \mathrm{t}=1.0 / 1.5 \mathrm{~ms}, \Delta \mathrm{f}=7 \mathrm{kHz}$ and a constant readout FA of $5^{\circ}$. 
In addition to ihMT-GRE images, all experiments included a sagittal 3D $\mathrm{T}_{1}$-weighted anatomical sequence (MPRAGE: TR/TE $=2300 / 3 \mathrm{~ms}$, inversion time $=900 \mathrm{~ms}$, readout bandwidth $=240 \mathrm{~Hz} /$ pixel, flip angle $=9^{\circ}$, matrix size of $256 \times 248 \times 176$ and isotropic voxel size of $1 \mathrm{~mm}$ ) as well as a sagittal interleaved multi-slice $\mathrm{rB}_{1}{ }^{+}$mapping sequence (pre-saturated turbo FLASH (19): $\mathrm{TR} / \mathrm{TE}=26290.0 / 2.24 \mathrm{~ms}$, readout bandwidth $=490 \mathrm{~Hz} /$ pixel, saturation flip angle $=80^{\circ}$, readout flip angle $=8^{\circ}$, in-plane matrix size of 96x96, 60 slices and reconstructed voxel size of $3 \times 3 \times 3 \mathrm{~mm}^{3}$ ).

\section{Image processing and statistical analyses}

MT-weighted magnitude images from all studies of each subject were combined in a stack and denoised using the MP-PCA routine from the MRtrix3 package (v. RC301) $(20,21)$, corrected for Gibbsringing artefacts with an isotropic 3D-cosine kernel (12), motion corrected (22) and combined to generate ihMTR maps according to eq. 1. An available pipeline comprising the aforementioned steps is available at: https://github.com/Isoustelle/ihmt proc (hash \#c9bb409). All ihMTR maps were rigidly co-registered onto their respective anatomical MPRAGE images using the Advanced Normalization Tools (ANTs; v2.0.1) (23).

Analyses focused on selected WM areas retrieved from the MNI white matter lobe atlas and WM regions of interest (ROI) from the JHU probabilistic atlas (24) through label propagation of the MNI template (symmetric ICBM 2009c) onto the anatomical MPRAGE volume using ANTs SyN (25). Deep grey matter (dGM) ROIs were also segmented using FreeSurfer's (v. 6.0.0) default recon-all pipeline on the MPRAGE image (26). These masks were eventually projected over the composite ihMTR images and $\mathrm{rB}_{1}{ }^{+}$maps linearly resampled at the MPRAGE resolution.

For Study 1, mean ihMTR values in WM lobes and in whole-WM were averaged across individuals and reported for all TR conditions. To evaluate the ihMTR response to $\mathrm{B}_{1}{ }^{+}$variations, the relative variations of ihMTR between nominal and deliberately attenuated $\mathrm{B}_{1}{ }^{+}$adjustments, $\delta_{\text {ihMTR, }}$ were computed voxelwise as:

$$
\delta_{\text {ihMTR }}=\frac{\operatorname{ihMTR}\left(V_{\text {ref, } 100 \%}\right)-\operatorname{ihMTR}\left(V_{\text {ref, } 80 \%}\right)}{\operatorname{ihMTR}\left(V_{\text {ref }, 100 \%}\right)}
$$

In addition, the RMS inMTR relative variation ( $\delta_{\text {ihMTR-RMS }}$ ) was also calculated:

$$
\delta_{\text {ihMTR-RMS }}=\sqrt{\frac{1}{N} \sum_{\nu}\left(\delta_{\text {ihMTR }}(\nu)\right)^{2}}(\text { eq. 5) }
$$


where $v$ is a voxel from the WM across all subjects (with a total of $\mathrm{N}$ voxels). This metric is indicative of the averaged dispersion due to the $-20 \% \mathrm{~B}_{1}{ }^{+}$variations deliberately induced by the decrease of the reference transmitter voltage.

$\delta_{\text {ihMtR }}$ values were extracted from voxels in the whole WM mask of each subject. In a second step all voxels were pooled according to their $\delta_{\text {ihMTR }}$ value in six different ranges in order to quantify the number of voxels within arbitrary error margins, defined as follows: $0 \%<\left|\delta_{\text {ihMTR }}\right|<5 \%$; $5 \%<\left|\delta_{\text {ihMTR }}\right|<10 \% ; \quad 10 \%<\left|\delta_{\text {ihMTR }}\right|<15 \% ; \quad 15 \%<\left|\delta_{\text {ihMTR }}\right|<20 \% ; \quad 20 \%<\left|\delta_{\text {ihMTR }}\right|<30 \% \quad$ and $30 \%<\left|\delta_{\text {ihMTR }}\right|<100 \%$.

To provide insight into the association of $\delta_{\text {ihMTR }}$ values with the actual distribution of $\mathrm{rB}_{1}{ }^{+}$in the $\mathrm{WM}$, voxels were also clustered into 31 equally-spaced classes of $\mathrm{rB}_{1}{ }^{+}$, spanning from the $2^{\text {nd }}$ to the $98^{\text {th }}$ percentile of its distribution for each subject, and the average $\delta_{\text {ihMTR }}$ was calculated for each cluster. For the purpose of comparison, $\delta_{\text {ihMTR }}$ was also simulated for each concentration scheme as a function of $\mathrm{rB}_{1}{ }^{+}$spanning from 0.90 to 1.15 .

For Study 2, the reproducibility of the test-retest protocol was assessed from the mean ihMTR values measured in ROIs of WM and deep GM derived from the JHU atlas and FreeSurfer segmentation as described above. The paired ihMTR and $\mathrm{rB}_{1}{ }^{+}$maps (test: ihMTR $\mathrm{A}_{\mathrm{A}}$ and $\mathrm{rB}_{1, \mathrm{~A}}{ }^{+}$; retest: ihMTR $\mathrm{B}_{\mathrm{B}}$ and $\mathrm{rB}_{1, \mathrm{~B}}{ }^{+}$) were rigidly co-registered onto the MPRAGE scan of the first session. Linear regression and BlandAltman analyses were performed between the ihMTR values derived from the two sessions.

\section{Results}

\section{Simulations}

Simulations of ihMTR as a function of $B_{1, R M S}{ }^{T R}$ (fixed $F A$ ) and FA (fixed $B_{1, R M S}{ }^{T R}$ ) are provided in Figure $2 a$ and $2 b$, respectively, for different TR values (i.e. different energy concentration schemes). At short TR values (TR $=38 \mathrm{~ms}$ ), ihMTR shows a monotonic increase with $B_{1, R M s}{ }^{\text {TR }}$ (Figure 2a) within the simulated range. We can perceive from this figure that $\mathrm{B}_{1}{ }^{+}$inhomogeneities, which will translate into variations around the nominal $B_{1, R M S}{ }^{T R}$ value, will result in a marked sensitivity of ihMTR to $B_{1, R M S}{ }^{T R}$ as illustrated by the slope of the curve around the nominal value of $3 \mu \mathrm{T}$. Conversely, as TR is lengthened to $350 \mathrm{~ms}$, the curve shows an inflection (for $B_{1, R M s}{ }^{\top R}>2 \mu \mathrm{T}$ ), that eventually leads to a maximum followed by a stable inMTR for strong RF saturation $\left(B_{1, R M S}{ }^{T R}>4 \mu \mathrm{T}\right)$. The moderately concentrated scheme $(T R=194 \mathrm{~ms})$ shows similarities with the TR = $350 \mathrm{~ms}$ scheme, with globally higher ihMTR for $B_{1, R M S}{ }^{T R}>2.3 \mu T$, and an inflexion and a saturation plateau both obtained for higher $B_{1, R M s}{ }^{T R}$ values. Focusing now on panel $b$, a decrease of inMTR with increasing FA is observed for all concentration schemes. Again, $\mathrm{B}_{1}{ }^{+}$inhomogeneities, which will also translate into variations of the actual $F A$, can 
modify the ihMTR value. Hence an antagonistic effect of the $\mathrm{B}_{1}{ }^{+}$-inhomogeneities can be understood from Figures $2 a$ and $2 b$. For instance, hyperintense $\mathrm{B}_{1}{ }^{+}$areas will tend to increase ihMTR through $B_{1, R M S}{ }^{T R}$ effects but also lead to a decrease of inMTR through the FA dependency.

The overall effects of the $B_{1}{ }^{+}$-inhomogeneities $\left(0.9<\mathrm{rB}_{1}{ }^{+}<1.1\right)$, through the joint modulation of $\mathrm{B}_{1, \mathrm{RMS}}{ }^{\mathrm{TR}}$ and $F A$, are illustrated in Figure $2 c$ as a function of TR for the nominal values of $B_{1, R M S}{ }^{T R}=3 \mu T$ and $F A=5^{\circ}$. IhMTRs increase rapidly at short TRs $(<100 \mathrm{~ms})$, then peak for moderate concentration schemes (TR in the range of 150 to $200 \mathrm{~ms}$ ) and slowly decay for longer TRs. Interestingly, the combined effects of $B_{1, R M S}{ }^{T R}$ and $F A$ variations around $3 \mu \mathrm{T}$ and $5^{\circ}$ allow creating conditions for robustness of ihMTR to $\mathrm{B}_{1}{ }^{+}$inhomogeneities for TR values between 350 and $402 \mathrm{~ms}$, as illustrated by the tightly clustered values of inMTR in this range.

\section{Experiments}

- Study 1: Variable TR at Fixed $B_{1, R M S}{ }^{T R}$

The shape and amplitude of the experimental curve describing ihMTR as a function of TR (Figure 3) were fairly close to the predicted ones for a nominal $B_{1, R M S}{ }^{T R}$ of $3 \mu \mathrm{T}$ (Figure $2 \mathrm{c}$ ) with values in lobar WM and in whole WM peaking at TR $=142 \mathrm{~ms}$. Of interest, ihMTR values measured for nominal $\mathrm{B}_{1}{ }^{+}$ and nominal $\mathrm{B}_{1}{ }^{+}$attenuated by $20 \%$ show progressive convergence beyond a TR of $194 \mathrm{~ms}$, and very close similarity for TR $=350 \mathrm{~ms}$ in the whole WM. This is further confirmed by the analysis considering the total number of WM voxels (Figure $4 \mathrm{~h}$ ), which shows that at TR $=350 \mathrm{~ms},\left|\delta_{\text {ihMTR }}\right|$ lies below $10-\%$ threshold for more than $73 \%$ of all WM voxels, whereas $\left|\delta_{\text {ihMTR }}\right|$ is below the $20-\%$ threshold for less than $15 \%$ of all WM voxels at TR $=38 \mathrm{~ms}$. $\delta_{\text {ihMTR-RMs }}$ scores, representing the RMS error over all WM voxels, emphasize the gain in performance as a monotonic decrease is observed with increasing TR, spanning from $28.7 \%(T R=38 \mathrm{~ms}$ ) to $9.1 \%(T R=350 \mathrm{~ms})$. A refined insight into the experimental robustness of ihMTR to $\mathrm{B}_{1}{ }^{+}$variations is provided in Figures $4 \mathrm{a}-\mathrm{g}$, showing the dependence of $\delta_{\text {inMTR }}$ on energy concentration (TR) for the clusters of WM voxels classified according to their actual $\mathrm{rB}_{1}{ }^{+}$ distribution. It appears that the RF energy concentration strategy obtained by lengthening the TR is most effective in brain areas for which the actual $B_{1, R M S}{ }^{T R}$ was stronger than the expected nominal value (i.e., $\mathrm{rB}_{1}{ }^{+} \geq 1.0$ ), again in line with simulations of $\delta_{\text {ihMTR }}$ variations with TR (Figures 4a-g; black solid lines).

Finally, a representative overview of the RF energy concentration effects on the ihMTR images is provided in Figure 5 . Whereas a short TR of $38 \mathrm{~ms}$ leads to a visually obvious bias, as evidenced by occipital-frontal and left-right gradients of ihMTR values matching the $\mathrm{rB}_{1}{ }^{+}$spatial profile, the ihMTR image obtained at $T R=350$ ms demonstrates a noticeably more homogeneous signal throughout the whole brain. 
- Study 2: Reproducibility

Test-retest reproducibility assessment yielded a very good agreement in linear regression between ihMTRs from experiments A and B (Figure 6; Pearson correlation coefficient, $\rho^{2}=0.977$; slope of 1.038 [p«0.001]; intercept of $-0.360 \%[p=0.001])$. Bland-Altman plot shows a close-to-zero bias $(-0.081 \%$ absolute ihMTR unit) and tight limits of agreement (0.535\%/-0.698\% absolute ihMTR unit). The coefficient of variation was low and amounted to $\mathrm{CoV}=0.027 \pm 0.028$ across all explored regions. Finally, no correlation ( $\rho^{2}=0.003$; Pearson) was found between residual differences of ihMTR (ihMTR $\mathrm{A}^{-}$ inMTR $\left.\mathrm{R}_{\mathrm{B}}\right)$ and $\mathrm{rB}_{1}{ }^{+}\left(\mathrm{rB}_{1, \mathrm{~A}}{ }^{+}-\mathrm{rB}_{1, \mathrm{~B}}{ }^{+}\right)$, emphasizing that discrepancies in test-retest ihMTR are not related to any $\mathrm{B}_{1}{ }^{+}$between-sessions differences.

\section{Discussion}

IhMTR has recently been validated as a semi-quantitative specific marker of myelination (2). In the context of clinical translation for application studies on various myelin-related diseases, it is of critical importance to minimize quantification biases that could affect the images' interpretation. For high field systems ( $B_{0} \geq 3 T$ ) especially, the RF excitation field shows an inhomogeneous distribution which may result in undesired variations of the ihMT signal over the investigated field of view. Minimizing the effect of $\mathrm{B}_{1}{ }^{+}$inhomogeneities on the ihMT signal is hence essential.

The current study aimed at evaluating the sensitivity of ihMTR measured with an ihMT-GRE sequence with various saturation preparation schemes to $\mathrm{B}_{1}{ }^{+}$variations occurring at $3 \mathrm{~T}$ in the human brain WM. This study was motivated by previous results obtained at $1.5 \mathrm{~T}$ (12) and guided by simulations. It was demonstrated that ihMT preparation where RF saturation energy is strongly concentrated in time is an efficient and reproducible strategy to mitigate the effects of $\mathrm{B}_{1}{ }^{+}$inhomogeneities on the WM signal measured at $3 \mathrm{~T}$ at the cost of a slight loss in sensitivity. More specifically ihMT-GRE with $B_{1, R M S}{ }^{T R}=3 \mu T$, $\mathrm{FA}=5^{\circ}$ and $\mathrm{TR}=350 \mathrm{~ms}$ resulted in relative variations of ihMTR lower than $10 \%$ in more than $70 \%$ of the total number of WM voxels following a global $\mathrm{B}_{1}{ }^{+}$reduction of $20 \%$. This configuration led to about $20-\%$ loss in sensitivity as shown by the variations of absolute ihMTR with TR $(\approx 8.5 \%$ for TR $=350 \mathrm{~ms}$ compared to a maximum value of $\approx 10.5 \%$ obtained for $T R=142 \mathrm{~ms}$, Figure 3 ).

\section{Optimal RF energy deposition: simulations versus experiments}

We used a recently proposed biophysical inMT model accounting for two nonzero $\mathrm{T}_{1 \mathrm{D}}$ components to simulate the ihMTR signal behavior in the realistic conditions of the ihMT-GRE sequence. The values of inMTR predicted by the model are in fair agreement with the experimental values (Figure $2 \mathrm{c}$ and Figure 3). The key to the lower sensitivity of ihMTR to $B_{1}{ }^{+}$variations lies in the inflection followed by a saturation of the curve describing the dependence of ihMTR on $B_{1, R M S}{ }^{T R}$ obtained at long TR values 
(Figure 2a), that is for high RF irradiation power during the RF pulses, leading to minimal ihMTR variations above a given RF power threshold. The underlying mechanisms of this specific behavior relates to the saturation of the RF-induced attenuation of the different magnetization pools for both single and dual offset experiments. Additional details are provided in Supporting Information along with plots of the steady state magnetization of all pools (Supporting Information Figure S1), and emphasize the strong impact of dipolar order on the saturation of the macromolecular pools.

The values of $\delta_{\text {ihMTR }}$, considered in this study as a quantitative measure of the insensitivity to $\mathrm{B}_{1}{ }^{+}$, also show very good agreement between simulations and experiments (Figure 4). Simulations were performed for fixed values representative of the WM tissue and selected from the literature $(14,18)$. However, accurate estimations of these parameters remain to be established in the human brain in vivo, and the values used in simulations only approximate the underlying biophysical processes. Differences in tissue parameters, which were not considered by the simulations, may thus explain the residual discrepancies between model predictions and experiments.

According to the simulation results, higher insensitivity to $\mathrm{B}_{1}{ }^{+}$could be achieved by concentrating even more the RF energy deposition using, for example, $B_{1, R M s}{ }^{T R}=3.0 \mu \mathrm{T}, \mathrm{FA}=5^{\circ}$ and $\mathrm{TR}=402 \mathrm{~ms}$ (Figure $2 \mathrm{c}$ ). Unfortunately, such RF energy concentration would require $B_{1 \text {,peak }}$ values incompatible with the hardware restrictions in the context of the proposed saturation scheme based on bursts of $N_{p}=8$ Tukey-shaped pulses.

An alternative strategy to increase the energy concentration would consist in increasing the number of pulses while shortening the interpulse delay by removing the spoiling gradient in between each saturation pulse. However, as attractive as this option sounds, it is not without problems because of increased sensitivity to direct saturation effects: the ihMTR response to the saturation offset frequency is greatly impacted by the absence of post-pulse gradient spoiler when using short duration pulses (PW $<1 \mathrm{~ms}$ ) and correlates with local $\mathrm{B}_{0}$ inhomogeneities (see Supporting information S2 and S3). An accurate description of the direct saturation phenomenon in short pulse ihMT experiments has yet to be developed, and advanced modeling using the configuration state formalism $(27,28)$ to comprehensively characterize the free pool behavior during an off-resonance irradiation and throughout the sequence is warranted.

Other strategies based on longer and fewer saturation pulses could reduce the demand of the spoiling gradients within the ihMT preparation. However, longer pulses imply longer pulse repetition times $(\Delta t)$, which reduce the sensitivity of the ihMT technique when using the frequency alternation approach for the dual-offset saturation (2): increases of $\Delta t$ result in ihMTR decreased because of marked signal attenuation of components with $T_{1 D}<\Delta t$ ( $T_{1 D}$-filtering). In contrast, cosine-modulated 
pulses do not filter short $T_{1 D} s$ components, hence enhancing the sensitivity of the technique, at the cost of a reduced specificity for myelinated tissues (2). Notwithstanding, this approach requires a $\sqrt{2}$ upscaling of the peak $B_{1}$ to reach identical energy deposition because of the loss of power due to the RF envelope modulation, which may limit its applicability. Overall, these two strategies for dual-offset saturation impart very different $\mathrm{T}_{1 \mathrm{D}}$-weighting $(2,29)$, and the choice of frequency modulation should primarily be based on the desired contrast.

\section{Robustness of ihMTR to $B_{1}{ }^{+}$inhomogeneity at $3 T$}

Ideally, the response of ihMTR to variations of $\mathrm{B}_{1}{ }^{+}$should have been assessed over the range of actual and local $\mathrm{B}_{1}{ }^{+}$values obtained in the human brain $\mathrm{WM}$ in vivo at $3 \mathrm{~T}$ (about $\pm 10 \%$ around the nominal value; see Figure 4a-g). However, since each voxel experiences a different local $\mathrm{B}_{1}{ }^{+}$intensity, the number of experimental conditions required to assess all voxels over the same actual $\mathrm{B}_{1}{ }^{+}$range and for multiple TR conditions would be prohibitive, especially in the context of in vivo experiments. Additionally, investigating $\mathrm{B}_{1}{ }^{+}$variations greater than the nominal value was not practically feasible because the maximum $B_{1 \text {,peak }}$ used in Study 1 (about $22.0 \mu \mathrm{T}$ ) was close to the maximum deliverable value. Hence, in the current study, we deliberately chose to induce a single and global $\mathrm{B}_{1}{ }^{+}$variation of $-20 \%$ which roughly corresponds to the total amplitude of the $\mathrm{B}_{1}{ }^{+}$variations measured in the whole brain WM, and thus could be considered as a worst-case experimental scenario. The proposed strategy may be considered as a case study providing a quantitative insight into how $\mathrm{B}_{1}{ }^{+}$variations may bias the inMTR metric. Although the results indicate that it is not possible to remove the bias induced by the global $20 \%$ drop in $\mathrm{B}_{1}{ }^{+}$in all voxels simultaneously, they clearly emphasized that configurations with strong RF energy concentration limit the relative ihMTR error below $10 \%$ for a global $20-\% \mathrm{~B}_{1}{ }^{+}$drop, especially in voxels for which $\mathrm{rB}_{1}^{+} \geq 1.0$.

Finally, the test-retest study further confirms that the reproducibility is not impacted by the RF field profile that may differ between two MRI sessions. A low intra-ROI variability (close-to-zero bias from the ROI-based Bland-Altman analysis) was observed, in line with previous results obtained at 1.5 T (12). Although $B_{1, R M S}{ }^{T R}$ was around $3 \mu \mathrm{T}$ in the current study performed at $3 \mathrm{~T}$ (i.e. much lower than the 5.4 $\mu T$ used in our previous $1.5 \mathrm{~T}$ study (12)), the ihMTR measured on healthy volunteers were relatively high, peaking around $10 \%$, as compared to the values measured at $1.5 \mathrm{~T}$ (maximum of around $16 \%$ in the pyramidal tract). This was made possible by optimizing the ihMT-GRE sequence in terms of dutycycle (i.e. moving towards longer TR), which additionally provides robustness to $\mathrm{B}_{1}{ }^{+}$inhomogeneities and benefits from the SNR efficiency at $3 \mathrm{~T}$, allowing for the generation of high-quality composite ihMTR maps. Overall, this proves the several interests of our proposed optimization for high-field protocols. Conversely, other groups working at $3 \mathrm{~T}$ have employed rather distributed ihMT-GRE 
sequences $(5,7,30)$, which impact both $\mathrm{SNR}$ and $\mathrm{B}_{1}{ }^{+}$sensitivity and hence potentially limits reproducibility.

\section{Alternative $B_{1}{ }^{+}$correction methods}

Recent studies have investigated alternative ways to tackle the $\mathrm{B}_{1}{ }^{+}$inhomogeneity effects on ihMT at $3 \mathrm{~T}$ with the use of the inverse ihMT ratio $(30,31)$ or ihMTsat $(32,33)$, two metrics that aim to remove biases from $\mathrm{T}_{1}$ and $\mathrm{B}_{1}{ }^{+}$effects and which are extensions of a strategy initially proposed for MT imaging (34). The calculation of inMTsat makes use of a modeling of the MT effects and readout exclusively considered at the free pool magnetization level. This strategy requires a model of the sequence events to describe the ihMT signal as a function of $\mathrm{T}_{1}$ and $\mathrm{B}_{1}{ }^{+}$. In addition, the $\mathrm{B}_{1}{ }^{+}$correction of ihMTsat requires to measure or simulate the actual dependence to $\mathrm{B}_{1}{ }^{+}$for given experimental conditions. In comparison, the ihMTR is model free and has the advantage of being simpler to derive.

\section{On the $B_{1, R M S} S^{T R}$ limitation due to SAR regulations and transmitter coil capabilities}

As most saturation-based methods, the SAR is a feature that limits the use of high energy pulses with regards to the ihMT technique. In this study, the maximum investigated $B_{1, R M S}{ }^{T R}(3.1 \mu T)$ corresponded to about $80 \%$ of the SAR regulatory limitation among the three subjects (with body weights spanning from 60 to $80 \mathrm{~kg}$ ) and close to the maximum deliverable $B_{1}$ peak amplitude. However, previous experiments on another 3T system (Verio, software version VB17, Siemens Healthineers, Erlangen, Germany) presented close-to-maximum SAR values for $B_{1, R M S}{ }^{T R}$ of only $2.7 \mu T$ among similar subjects. Explorable energy concentration schemes for a targeted population are therefore subject to a compromise between SAR regulations and available maximum $B_{1}$ peak amplitude which both depend on the transmitter coil and the actual load of tissues within the coil. Local brain transmit coils may help improving the compromise that was reached in this study using a body coil transmission. Notably, the partial Fourier saturation method did help increasing the effective $B_{1, R M S}{ }^{T R}$ by reducing the energy deposition in the k-space periphery (12). With our available average power, we showed the possibility of concentrating RF energy enough such that a reasonable immunity to $\mathrm{B}_{1}{ }^{+}$variations can be obtained in a clinical setting. Overall, the capacity to work at high $B_{1, R M S}{ }^{T R}$ would offer additional flexibility to achieve sufficient RF energy concentration in order to reach robust regimes of ihMTR to $B_{1}^{+}$ inhomogeneities.

\section{Limitations}

Apart from the reproducibility experiments, this study was performed on only six different subjects divided into two experimental groups. Individuals naturally present different anatomy and physiology, 
and hence, potential differences in WM content, impacting ihMTR absolute values and response to $B_{1}{ }^{+}$ variations, as observed in Figure 4 . In addition, the $\mathrm{B}_{1}{ }^{+}$cluster analysis may be slightly inaccurate due to head movement throughout the protocol, yielding inaccuracies when relating ihMTR and $\mathrm{B}_{1}{ }^{+}$maps, such as voxels shifting from classes to classes of $\mathrm{rB}_{1}{ }^{+}$. However, considering these movements as low and assuming a $\mathrm{B}_{1}{ }^{+}$profile slowly varying across space, such effect should have minor impact in analyses given the large number of voxels involved in this investigation (more than 246,000 per subject in average), and did not preclude to observe experimentally the trends predicted by simulations.

\section{Conclusion}

This study has provided a thorough analysis of the sensitivity of ihMTR to $\mathrm{B}_{1}{ }^{+}$variations typically observed in the human WM at 3 T. A strategy was proposed to minimize the bias induced by $\mathrm{B}_{1}{ }^{+}$ inhomogeneities within the imposed hardware and SAR regulatory constraints. Although the most robust settings to $\mathrm{B}_{1}{ }^{+}$variations lead to a loss in sensitivity, the proposed protocol provided confidence that inMTR measured over the whole WM suffers from minimal bias so that the observed variations would reflect biophysical mechanisms. The most robust setting was assessed in a reproducibility study and showed excellent accuracy compatible with clinical studies.

\section{Acknowledgements}

The authors thank V. Gimenez, P. Viout, L. Pini and C. Costes for technical support and management.

\section{References}

1. Varma G, Duhamel G, de Bazelaire C, Alsop DC. Magnetization transfer from inhomogeneously broadened lines: A potential marker for myelin. Magn. Reson. Med. 2015;73:614-622 doi: 10.1002/mrm.25174.

2. Duhamel G, Prevost VH, Cayre M, et al. Validating the sensitivity of inhomogeneous magnetization transfer (ihMT) MRI to myelin with fluorescence microscopy. Neuroimage 2019;199:289-303 doi: 10.1016/j.neuroimage.2019.05.061.

3. Varma G, Girard OM, Prevost VH, Grant AK, Duhamel G, Alsop DC. Interpretation of magnetization transfer from inhomogeneously broadened lines (ihMT) in tissues as a dipolar order effect within motion restricted molecules. J. Magn. Reson. 2015;260:67-76 doi: 10.1016/j.jmr.2015.08.024.

4. Varma G, Girard OM, Prevost VH, Grant AK, Duhamel G, Alsop DC. In vivo measurement of a new source of contrast, the dipolar relaxation time, T $1 \mathrm{D}$, using a modified inhomogeneous magnetization transfer (ihMT) sequence. Magn. Reson. Med. 2017;78:1362-1372 doi: $10.1002 / \mathrm{mrm} .26523$.

5. Ercan E, Varma G, Mädler B, et al. Microstructural correlates of 3D steady-state inhomogeneous magnetization transfer (ihMT) in the human brain white matter assessed by myelin water imaging 
and diffusion tensor imaging. Magn. Reson. Med. 2018;80:2402-2414 doi: 10.1002/mrm.27211.

6. Ercan E, Varma G, Dimitrov IE, et al. Combining inhomogeneous magnetization transfer and multipoint Dixon acquisition: Potential utility and evaluation. Magn. Reson. Med. 2020:mrm.28571 doi: $10.1002 / \mathrm{mrm} .28571$.

7. Zhang L, Chen T, Tian H, et al. Reproducibility of inhomogeneous magnetization transfer (ihMT): A test-retest, multi-site study. Magn. Reson. Imaging 2019;57:243-249 doi: 10.1016/j.mri.2018.11.010.

8. Van Obberghen E, Mchinda S, le Troter A, et al. Evaluation of the Sensitivity of Inhomogeneous Magnetization Transfer (ihMT) MRI for Multiple Sclerosis. Am. J. Neuroradiol. 2018;39:634-641 doi: 10.3174/ajnr.A5563.

9. Girard OM, Callot V, Prevost VH, et al. Magnetization transfer from inhomogeneously broadened lines (ihMT): Improved imaging strategy for spinal cord applications. Magn. Reson. Med.

2017;77:581-591 doi: 10.1002/mrm.26134.

10. Girard OM, Prevost VH, Varma G, Cozzone PJ, Alsop DC, Duhamel G. Magnetization transfer from inhomogeneously broadened lines (ihMT): Experimental optimization of saturation parameters for human brain imaging at 1.5 Tesla. Magn. Reson. Med. 2015;73:2111-2121 doi: 10.1002/mrm.25330.

11. Malik SJ, Teixeira RPAG, West DJ, Wood TC, Hajnal J V. Steady-state imaging with inhomogeneous magnetization transfer contrast using multiband radiofrequency pulses. Magn. Reson. Med. 2019:mrm.27984 doi: 10.1002/mrm.27984.

12. Mchinda S, Varma G, Prevost VH, et al. Whole brain inhomogeneous magnetization transfer (ihMT) imaging: Sensitivity enhancement within a steady-state gradient echo sequence. Magn. Reson. Med. 2018;79:2607-2619 doi: 10.1002/mrm.26907.

13. Varma G, Girard OM, Mchinda S, et al. Low duty-cycle pulsed irradiation reduces magnetization transfer and increases the inhomogeneous magnetization transfer effect. J. Magn. Reson. 2018;296:60-71 doi: 10.1016/j.jmr.2018.08.004.

14. Carvalho VND, Hertanu A, Grélard A, et al. MRI assessment of multiple dipolar relaxation time ( $T$ 1D) components in biological tissues interpreted with a generalized inhomogeneous magnetization transfer (ihMT) model. J. Magn. Reson. 2020;311:106668 doi: 10.1016/j.jmr.2019.106668.

15. Harris FJ. On the use of windows for harmonic analysis with the discrete Fourier transform. Proc. IEEE 1978;66:51-83 doi: 10.1109/PROC.1978.10837.

16. Mchinda S, Varma G, Draveny R, et al. Whole brain inhomogeneous Magnetization Transfer (ihMT) imaging at 3T: concentrating RF power to mitigate RF inhomogeneities effects. In:

Proceedings 26th Scientific Meeting, International Society for Magnetic Resonance in Medicine. ; 2018. p. 786.

17. Soustelle L, Mchinda S, Troalen T, et al. Assessment of the sensitivity of inhomogeneous Magnetization Transfer (ihMT) brain imaging with B1+ variations at 3T. In: Proceedings of the 28th Annual Meeting of ISMRM. Virtual meeting; 2020. p. 3167.

18. Yarnykh VL. Fast macromolecular proton fraction mapping from a single off-resonance magnetization transfer measurement. Magn. Reson. Med. 2012;68:166-178 doi: 10.1002/mrm.23224.

19. Chung S, Kim D, Breton E, Axel L. Rapid B1+ mapping using a preconditioning RF pulse with TurboFLASH readout. Magn. Reson. Med. 2010;64:n/a-n/a doi: 10.1002/mrm.22423.

20. Veraart J, Novikov DS, Christiaens D, Ades-aron B, Sijbers J, Fieremans E. Denoising of diffusion MRI using random matrix theory. Neuroimage 2016;142:394-406 doi: 
10.1016/j.neuroimage.2016.08.016.

21. Grussu F, Battiston M, Veraart J, et al. Multi-parametric quantitative in vivo spinal cord MRI with unified signal readout and image denoising. Neuroimage 2020;217:116884 doi: 10.1016/j.neuroimage.2020.116884.

22. Soustelle L, Lamy J, Le Troter A, et al. A Motion Correction Strategy for Multi-Contrast based 3D parametric imaging : Application to Inhomogeneous Magnetization Transfer (ihMT). bioRxiv 2020 doi: 10.1101/2020.09.11.292649.

23. Avants BB, Tustison N, Song G. Advanced Normalization Tools (ANTS). Insight J. 2 2009:1-35.

24. Wakana S, Caprihan A, Panzenboeck MM, et al. Reproducibility of quantitative tractography methods applied to cerebral white matter. Neuroimage 2007;36:630-644 doi:

10.1016/j.neuroimage.2007.02.049.

25. Avants BB, Tustison NJ, Song G, Cook PA, Klein A, Gee JC. A reproducible evaluation of ANTs similarity metric performance in brain image registration. Neuroimage 2011;54:2033-2044 doi: 10.1016/j.neuroimage.2010.09.025.

26. Dale AM, Fischl B, Sereno MI. Cortical Surface-Based Analysis. Neuroimage 1999;9:179-194 doi: 10.1006/nimg.1998.0395.

27. Weigel M. Extended phase graphs: Dephasing, RF pulses, and echoes - pure and simple. J. Magn. Reson. Imaging 2015;41:266-295 doi: 10.1002/jmri.24619.

28. Malik SJ, Teixeira RPAG, Hajnal J V. Extended phase graph formalism for systems with magnetization transfer and exchange. Magn. Reson. Med. 2018;80:767-779 doi:

10.1002/mrm.27040.

29. Prevost VH, Girard OM, Mchinda S, Varma G, Alsop DC, Duhamel G. Optimization of inhomogeneous magnetization transfer (ihMT) MRI contrast for preclinical studies using dipolar relaxation time ( T 1D ) filtering. NMR Biomed. 2017;30:e3706 doi: 10.1002/nbm.3706.

30. Wood TC, Damestani NL, Lawrence AJ, et al. Silent myelin-weighted magnetic resonance imaging. Wellcome Open Res. 2020;5:74 doi: 10.12688/wellcomeopenres.15845.1.

31. Geeraert BL, Lebel RM, Mah AC, et al. A comparison of inhomogeneous magnetization transfer, myelin volume fraction, and diffusion tensor imaging measures in healthy children. Neuroimage 2018;182:343-350 doi: 10.1016/j.neuroimage.2017.09.019.

32. Munsch F, Varma G, Taso M, et al. Characterization of the cortical myeloarchitecture with inhomogeneous magnetization transfer imaging (ihMT). Neuroimage 2021;225:117442 doi: 10.1016/j.neuroimage.2020.117442.

33. Rowley CD, Campbell JSW, Wu Z, et al. A Model-based Framework for Correcting B1+ Inhomogeneity Effects in Magnetization Transfer Saturation and Inhomogeneous Magnetization Transfer Saturation Maps. Magn. Reson. Med. 2021;In Review:1-16 doi: 10.1002/mrm.28831.

34. Helms G, Dathe H, Kallenberg K, Dechent P. High-resolution maps of magnetization transfer with inherent correction for RF inhomogeneity and T 1 relaxation obtained from 3D FLASH MRI. Magn. Reson. Med. 2008;60:1396-1407 doi: 10.1002/mrm.21732. 


\section{Figure and Table captions}

Table 1: Experimental parameters of the ihMT-GRE sequences. Common saturation parameters were: bursts of $\mathrm{N}_{\mathrm{p}}=8$ Tukey-shaped saturation pulses $(r=0.3)$ with a gradient spoiler after each off-resonance pulse, $\mathrm{PW} / \Delta \mathrm{t}=1.0 / 1.5 \mathrm{~ms}, \Delta \mathrm{f}=7 \mathrm{kHz}$ and a constant readout flip angle of $5^{\circ}$.

Figure 1: ihMT-GRE pulse sequence with different RF energy deposition schemes (corresponding to different RF saturation duty-cycles). Bursts of $\mathrm{N}_{\mathrm{p}}$ off-resonance saturation pulses are followed by GRE readout segment(s). The use of a short TR (a) results in a rather distributed RF energy deposition over the repetition time, whereas a long TR (b) results in a more concentrated RF energy deposition. Multiple readout segments $\left(\mathrm{N}_{\mathrm{GRE}}\right)$ were used with increased TR to improve the data sampling efficiency and reduce the total acquisition time.

Figure 2: Simulations of ihMTR dependence on $B_{1, R M S}{ }^{T R}(a)$ and readout flip angles $(b)$ for varying energy concentration schemes corresponding to distributed ( $T R=38 \mathrm{~ms}$ ), intermediate (TR=194 ms) and concentrated (TR=350 ms) RF energy deposition. IhMTR as a function of TR (c), performed for nominal $B_{1, R M S}{ }^{\top R}=3 \mu$ T and readout flip angle of $5^{\circ}$, are plotted for $\mathrm{rB}_{1}{ }^{+}$of 0.9 (blue), 1.0 (black) and 1.1 (red). Dashed grey lines indicate the working nominal power as used in experiments $(3 \mu \mathrm{T})$ or readout flip angle $\left(5^{\circ}\right) . \mathrm{TR}_{\text {sub }}$ was maintained constant $(6.5 \mathrm{~ms})$ for all simulations.

Figure 3: Mean values of ihMTR evaluated in WM lobes and in whole WM as a function of the RF energy concentration (increasing TR) averaged across all subjects. Filled and open markers correspond to ihMTR measured at nominal $\mathrm{B}_{1}{ }^{+}$and nominal $\mathrm{B}_{1}{ }^{+}$attenuated by $20 \%$, respectively.

Figure 4: Mean measured $\delta_{\text {ihMTR }}$ values in WM voxels clustered according to their $\mathrm{rB}_{1}{ }^{+}$distribution for each subject and different energy concentration schemes (TR; a-g). The individual markers' sizes are proportional to the number of voxels in a cluster to provide an indication of the global $\mathrm{rB}_{1}{ }^{+}$distribution in the WM. Panel g: Fraction of the total voxels in WM pooled according to $\left|\delta_{\text {inMTR }}\right|$ thresholds $(0,5$, $10,15,20,30$ and 100\%) across all subjects and along the investigated energy concentration schemes $(T R) ; \delta_{\text {ihMTR-RMs }}$ values are reported (top). Dashed horizontal lines in panels a-g indicate $\left|\delta_{\text {ihMTR }}\right|$ thresholds with the same color code as that of panel g.

Figure 5: Representative $\mathrm{rB}_{1}{ }^{+}$(top row) and ihMTR views obtained with distributed (TR=38 ms, middle row) and concentrated ( $T R=350 \mathrm{~ms}$, bottom row) energy concentration (nominal $\mathrm{B}_{1, \mathrm{RMS}}{ }^{\mathrm{TR}}=3 \mu \mathrm{T}$ ). White arrows indicate remarkable areas where a drop or an increase in $\mathrm{rB}_{1}{ }^{+}$resulted in a drop or an increase in ihMTR values for TR=38 ms. Conversely, ihMTR maps at TR=350 ms exhibit greatly reduced sensitivity to $\mathrm{rB}_{1}{ }^{+}$variations. 
Figure 6: Reproducibility of inMT-GRE at $T R=350$ ms and $B_{1, R M s}{ }^{T R}=3 \mu T$. (a) Linear regression (line of unity: solid; regression line: dashed) and (b) Bland-Altman (LOA: limits of agreement; CoV: coefficient of variation) plots of inMTR values in WM regions (black) and deep GM (grey) derived from the testretest (A-B) experiments. (c) Representative orthogonal views of a 2-mm isotropic ihMTR map.

\section{Supporting Information}

Additional Supporting Information may be found in the online version of this article.

Figure S1: Simulations of normalized longitudinal magnetizations in steady-state of the free pool (left column) and bound pools (long $\mathrm{T}_{1 \mathrm{D}}$ : middle column; short $\mathrm{T}_{1 \mathrm{D}}$ : right column) in the single MT (top row) and dual MT (bottom row) experiments as a function of $B_{1, R M S}{ }^{T R}$ and for TR of 38, 194 and 350 ms. Dashed grey lines indicate the targeted nominal power as used in experiments $(3 \mu \mathrm{T})$. Simulated tissue parameters: $R_{1, f} / R_{1, b}=1.0 / 1.0 \mathrm{~s}^{-1}, T_{2, f}=22.0 \mathrm{~ms}, T_{2, b}=10.0 \mu \mathrm{s}, \mathrm{R}=19.0 \mathrm{~s}^{-1}, \mathrm{M}_{0, \mathrm{f}} / \mathrm{M}_{0, \mathrm{~b}}=1.0 / 0.16, \mathrm{~T}_{1 \mathrm{D}, 1} / \mathrm{T}_{1 \mathrm{D}, 2}$ $=10.0 / 0.4 \mathrm{~ms}$ and $\mathrm{f}_{\mathrm{D}}=0.5$.

Figure S2: ihMTR maps as a function of the preparation pulses offset frequency, generated with $P W / \Delta t=1.0 / 1.5 \mathrm{~ms}\left(\mathrm{~N}_{\mathrm{p}}=8\right)$ with post-pulse spoiling gradients (top) and $\mathrm{PW} / \Delta \mathrm{t}=0.5 / 0.75 \mathrm{~ms}\left(\mathrm{~N}_{\mathrm{p}}=16\right)$ with a single post-burst spoiling gradient (bottom; note the changes in image dynamic), and corresponding $\Delta \mathrm{f}_{0}$ map. Common ihMT sequence parameters: $B_{1, \text { peak }}=22.0 \mu \mathrm{T}, \mathrm{B}_{1, \mathrm{RMs}}{ }^{\mathrm{TR}}=3.0 \mu \mathrm{T}, \mathrm{TR}=350 \mathrm{~ms}, \mathrm{TR}_{\text {sub }}=6.5$ $\mathrm{ms}$, number of GRE segments $=52$, readout flip angle $=5^{\circ}$.

Figure S3: Equivalent on-resonance flip angle of a single off-resonance Tukey-shaped $(r=0.3$, $B_{1, \text { peak }}=22.0 \mu \mathrm{T}$ ) pulse as a function of its frequency offset for $\mathrm{PW}=1.0 \mathrm{~ms}$ (red) and $\mathrm{PW}=0.5 \mathrm{~ms}$ (black). Curves were generated by simulations of the Bloch equations, and calculated from the longitudinal magnetization at the end of the pulse application as $\cos ^{-1}\left(M_{2} / M_{0}\right)$ (starting from an equilibrium state $M_{2}=M_{0}$, and neglecting relaxation and $M T$ effects). Dashed gray lines indicate experimental offresonance frequencies (Fig. S2). 
Table 1: Experimental parameters of the ihMT-GRE sequences. Common saturation parameters were: bursts of $N_{p}=8$ Tukey-shaped saturation pulses ( $\left.r=0.3\right)$ with a gradient spoiler after each off-resonance pulse, $P W / \Delta t=1.0 / 1.5 \mathrm{~ms}, \Delta f=7 \mathrm{kHz}$ and a constant readout flip angle of $5^{\circ}$.

\begin{tabular}{|c|c|c|c|c|c|c|c|c|c|}
\hline Experiments & $\begin{array}{c}\mathrm{B}_{1, \mathrm{RMM}} \mathrm{T}^{\mathrm{TR}} \\
(\mu \mathrm{T})\end{array}$ & TR (ms) & $\begin{array}{c}\text { Saturation } \\
\text { pulse } B_{1} \\
\text { peak }(\mu \mathrm{T})\end{array}$ & $\begin{array}{l}\text { Number of } \\
\text { readout } \\
\text { segments } \\
\text { per TR }\end{array}$ & $\begin{array}{c}\mathrm{TR}_{\text {sub }} / \mathrm{TE} \\
(\mathrm{ms})\end{array}$ & $\begin{array}{c}\text { Readout } \\
\text { bandwidth } \\
\text { (Hz/voxel) }\end{array}$ & $\begin{array}{l}\text { Resolution } \\
(\mathrm{mm})\end{array}$ & Matrix size & $\begin{array}{l}\text { Acquisition } \\
\text { time }\end{array}$ \\
\hline 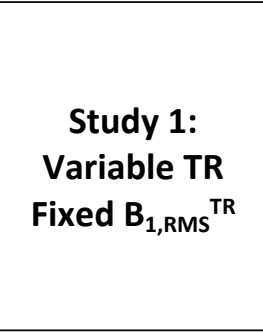 & 3.0 & $\begin{array}{c}38 \\
90 \\
142 \\
194 \\
246 \\
298 \\
350 \\
\end{array}$ & \begin{tabular}{c|}
7.3 \\
11.1 \\
14.0 \\
16.4 \\
18.4 \\
20.3 \\
22.0 \\
\end{tabular} & $\begin{array}{c}4 \\
12 \\
20 \\
28 \\
36 \\
44 \\
52 \\
\end{array}$ & $6.5 / 3.0$ & 200 & 2.5 iso. & $96 \times 96 \times 72$ & $\begin{array}{l}6^{\prime} 14^{\prime \prime} \\
5^{\prime} 04^{\prime \prime} \\
4^{\prime} 52^{\prime \prime} \\
4^{\prime} 44^{\prime \prime} \\
4^{\prime} 43^{\prime \prime} \\
4^{\prime} 38^{\prime \prime} \\
4^{\prime} 36^{\prime \prime}\end{array}$ \\
\hline $\begin{array}{c}\text { Study 2: } \\
\text { Reproducibility }\end{array}$ & 3.0 & 350 & 22.0 & 52 & $6.5 / 2.9$ & 210 & 2.0 iso. & $128 \times 128 \times 88$ & 9'35" \\
\hline
\end{tabular}


(a) Distributed energy deposition
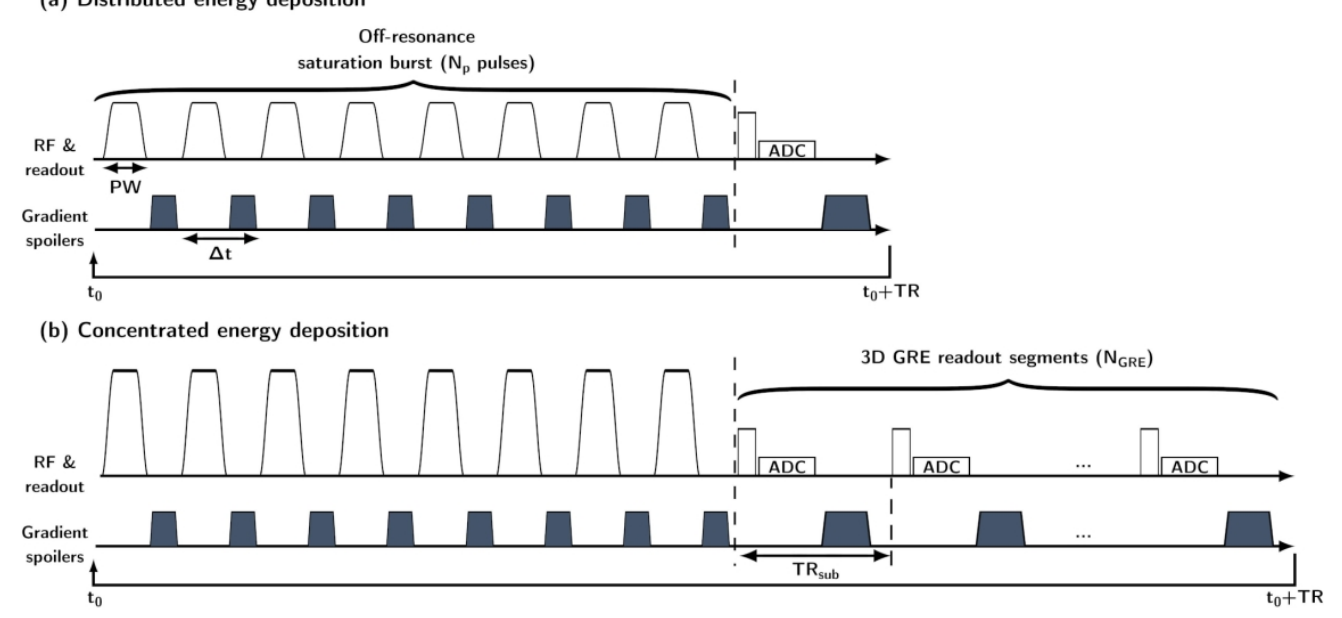

Figure 1: ihMT-GRE pulse sequence with different RF energy deposition schemes (corresponding to different RF saturation duty-cycles). Bursts of $\mathrm{N}_{\mathrm{p}}$ off-resonance saturation pulses are followed by GRE readout segment(s). The use of a short TR (a) results in a rather distributed RF energy deposition over the repetition time, whereas a long TR (b) results in a more concentrated RF energy deposition. Multiple readout segments $\left(\mathrm{N}_{\mathrm{GRE}}\right)$ were used with increased TR to improve the data sampling efficiency and reduce the total acquisition time.

$175 \times 86 \mathrm{~mm}(600 \times 600 \mathrm{DPI})$ 

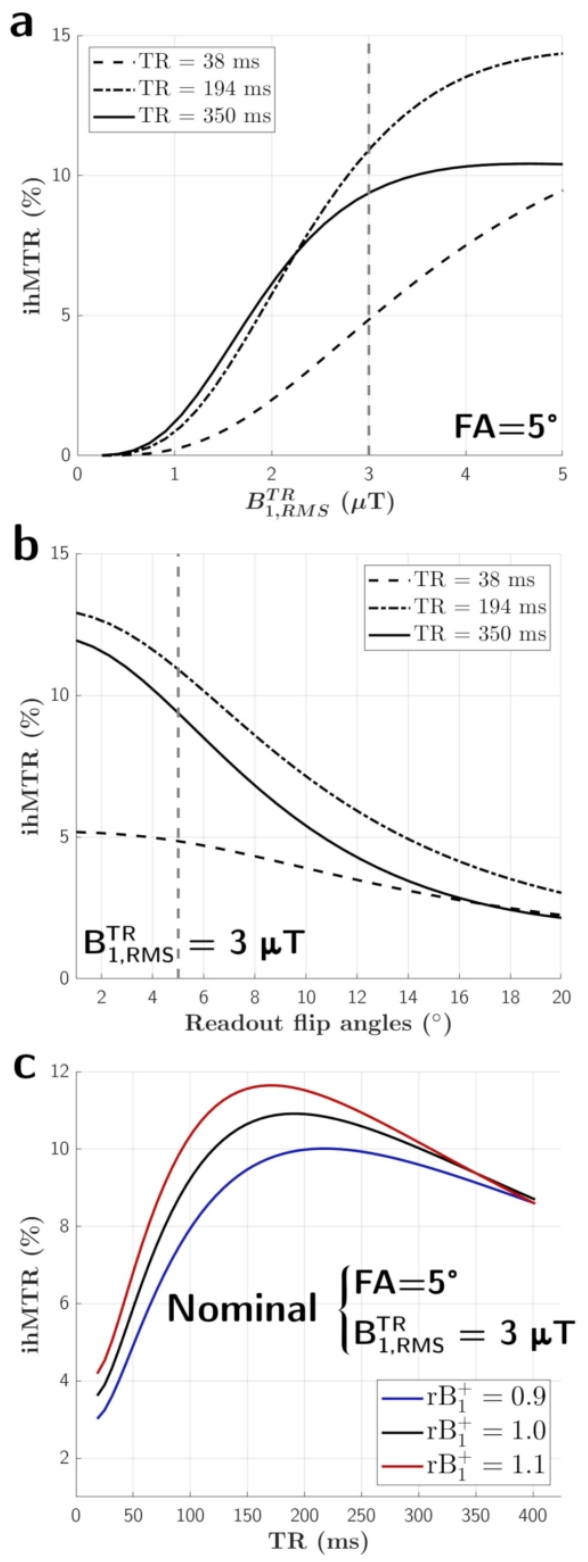

Figure 2: Simulations of ihMTR dependence on $B_{1, R M S}{ }^{T R}$ (a) and readout flip angles (b) for varying energy concentration schemes corresponding to distributed ( $T R=38 \mathrm{~ms}$ ), intermediate ( $T R=194 \mathrm{~ms}$ ) and concentrated ( $T R=350 \mathrm{~ms}$ ) RF energy deposition. IhMTR as a function of TR (c), performed for nominal $\mathrm{B}_{1, \mathrm{RMS}}{ }^{\mathrm{TR}}=3 \mu \mathrm{T}$ and readout flip angle of $5^{\circ}$, are plotted for $\mathrm{rB}_{1}{ }^{+}$of 0.9 (blue), 1.0 (black) and 1.1 (red). Dashed grey lines indicate the working nominal power as used in experiments $(3 \mu \mathrm{T})$ or readout flip angle $\left(5^{\circ}\right) . \mathrm{TR}_{\text {sub }}$ was maintained constant $(6.5 \mathrm{~ms})$ for all simulations.

$$
86 \times 215 \mathrm{~mm}(600 \times 600 \mathrm{DPI})
$$




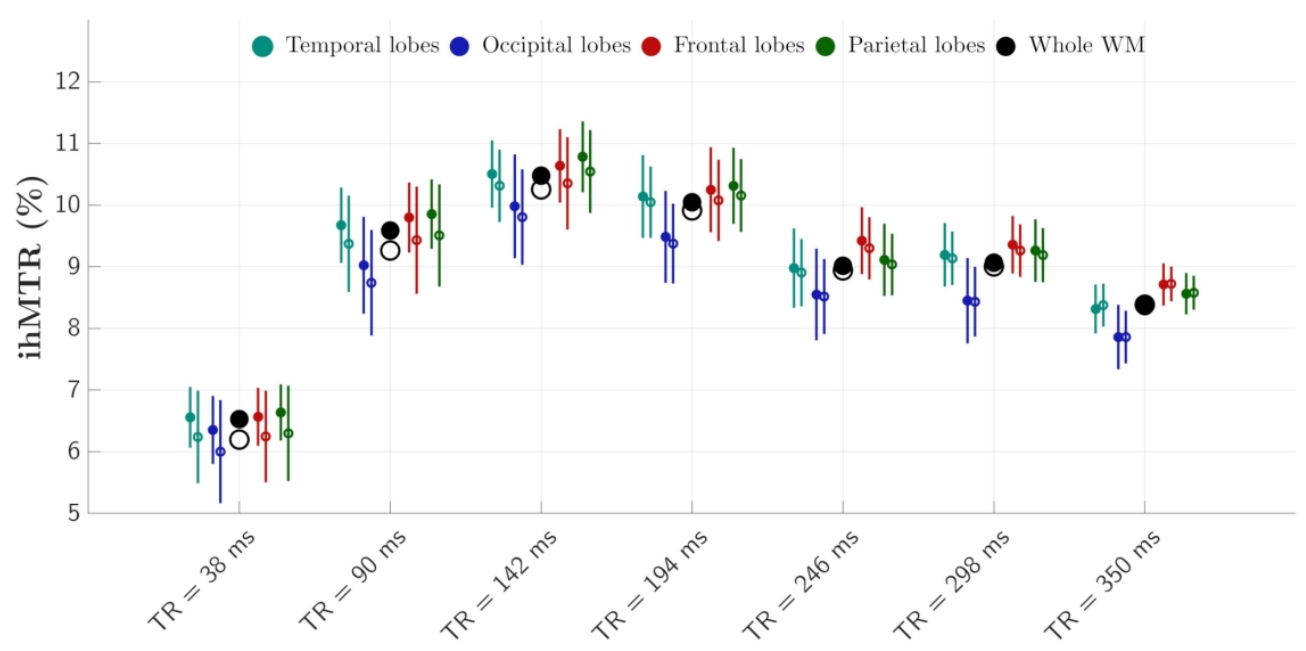

Figure 3: Mean values of ihMTR evaluated in WM lobes and in whole WM as a function of the RF energy concentration (increasing TR) averaged across all subjects. Filled and open markers correspond to ihMTR measured at nominal $\mathrm{B}_{1}{ }^{+}$and nominal $\mathrm{B}_{1}{ }^{+}$attenuated by $20 \%$, respectively.

\section{$175 \times 87 \mathrm{~mm}(600 \times 600 \mathrm{DPI})$}



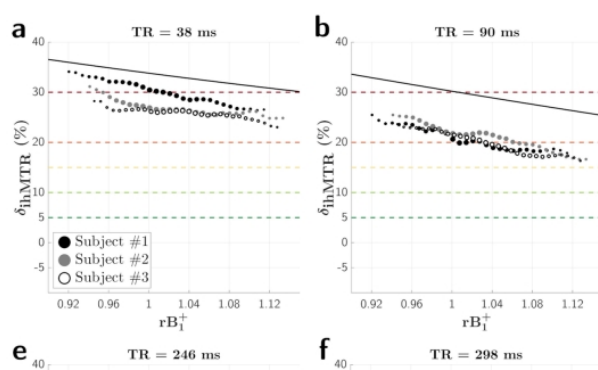

$\mathbf{d}_{40} \quad \mathrm{TR}=194 \mathrm{~ms}$
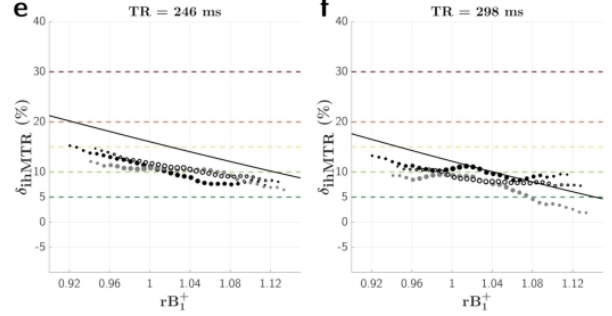
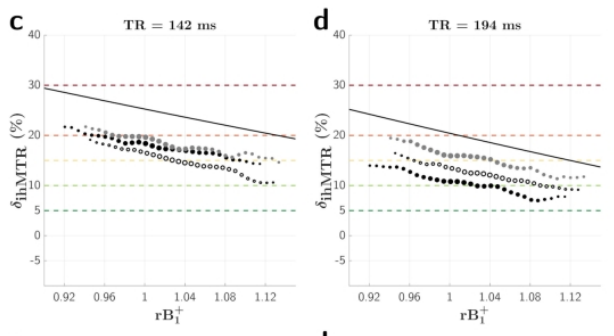

$\mathbf{g}_{40 .} \quad \mathrm{TR}=350 \mathrm{~ms}$

h

h
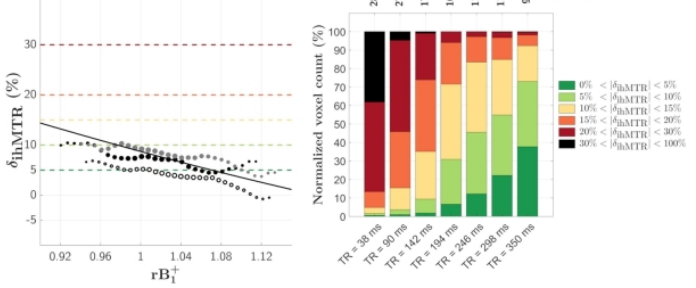

Figure 4: Mean measured $\delta_{\text {ihMTR }}$ values in WM voxels clustered according to their $\mathrm{rB}_{1}{ }^{+}$distribution for each subject and different energy concentration schemes (TR; a-g). The individual markers' sizes are proportional to the number of voxels in a cluster to provide an indication of the global $\mathrm{rB}_{1}{ }^{+}$distribution in the WM. Panel

$\mathrm{g}$ : Fraction of the total voxels in WM pooled according to $\left|\delta_{\text {ihMTR }}\right|$ thresholds $(0,5,10,15,20,30$ and $100 \%$ ) across all subjects and along the investigated energy concentration schemes (TR); $\delta_{\text {ihMTR-RMS }}$ values are reported (top). Dashed horizontal lines in panels a-g indicate $\left|\delta_{\text {ihMTR }}\right|$ thresholds with the same color code as that of panel $\mathrm{g}$.

$241 \times 120 \mathrm{~mm}(300 \times 300$ DPI $)$ 
Figure 5: Representative $\mathrm{rB}_{1}{ }^{+}$(top row) and ihMTR views obtained with distributed ( $\mathrm{TR}=38$ ms, middle row) and concentrated ( $T R=350 \mathrm{~ms}$, bottom row) energy concentration (nominal $B_{1, R M S}{ }^{T R}=3 \mu T$ ). White arrows indicate remarkable areas where a drop or an increase in $\mathrm{rB}_{1}{ }^{+}$resulted in a drop or an increase in ihMTR values for $T R=38$ ms. Conversely, ihMTR maps at $T R=350$ ms exhibit greatly reduced sensitivity to $r_{1}{ }^{+}$ variations.

$175 \times 106 \mathrm{~mm}(300 \times 300$ DPI $)$ 

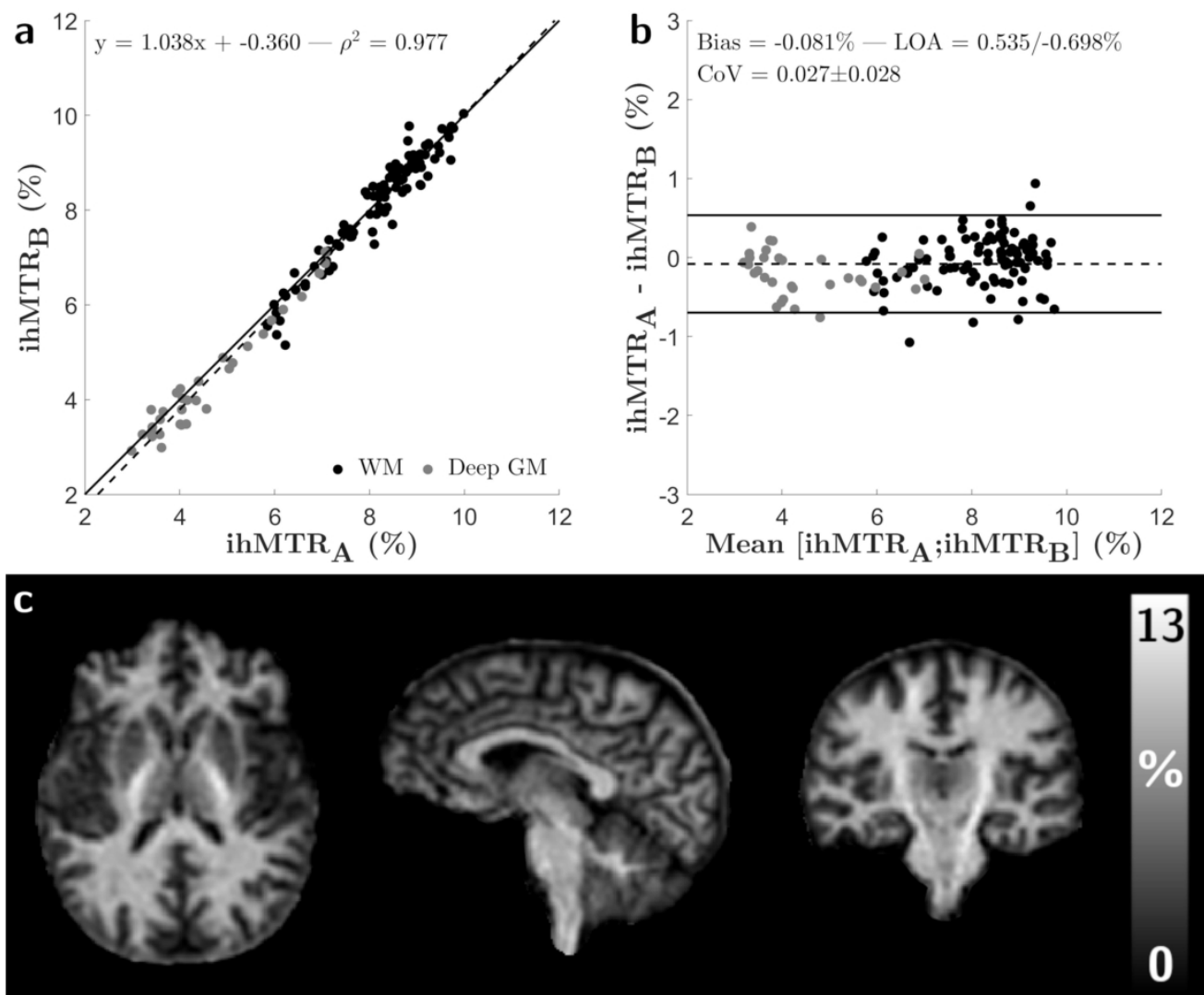

Figure 6: Reproducibility of ihMT-GRE at $T R=350 \mathrm{~ms}$ and $B_{1, R M S}{ }^{T R}=3 \mu T$. (a) Linear regression (line of unity: solid; regression line: dashed) and (b) Bland-Altman (LOA: limits of agreement; CoV: coefficient of variation) plots of inMTR values in WM regions (black) and deep GM (grey) derived from the test-retest (AB) experiments. (c) Representative orthogonal views of a 2-mm isotropic ihMTR map.

$175 \times 147 \mathrm{~mm}(300 \times 300 \mathrm{DPI})$ 


\section{A strategy to reduce the sensitivity of inhomogeneous Magnetization Transfer (ihMT) imaging to radiofrequency transmit field variations at $3 \mathrm{~T}$}

Lucas Soustelle ${ }^{1,2}$, Thomas Troalen ${ }^{3}$, Andreea Hertanu ${ }^{1,2}$, Samira Mchinda ${ }^{1,2}$, Jean-Philippe Ranjeva $^{1,2}$, Maxime Guye ${ }^{1,2}$, Gopal Varma ${ }^{4}$, David C. Alsop ${ }^{4}$, Guillaume Duhamel ${ }^{1,2}$, Olivier M. Girard ${ }^{1,2}$

1. Aix Marseille Univ, CNRS, CRMBM, Marseille, France

2. APHM, Hôpital Universitaire Timone, CEMEREM, Marseille, France

3. Siemens Healthcare SAS, Saint-Denis, France

4. Division of MR Research, Radiology, Beth Israel Deaconess Medical Center, Harvard Medical School, Boston, MA, United States 

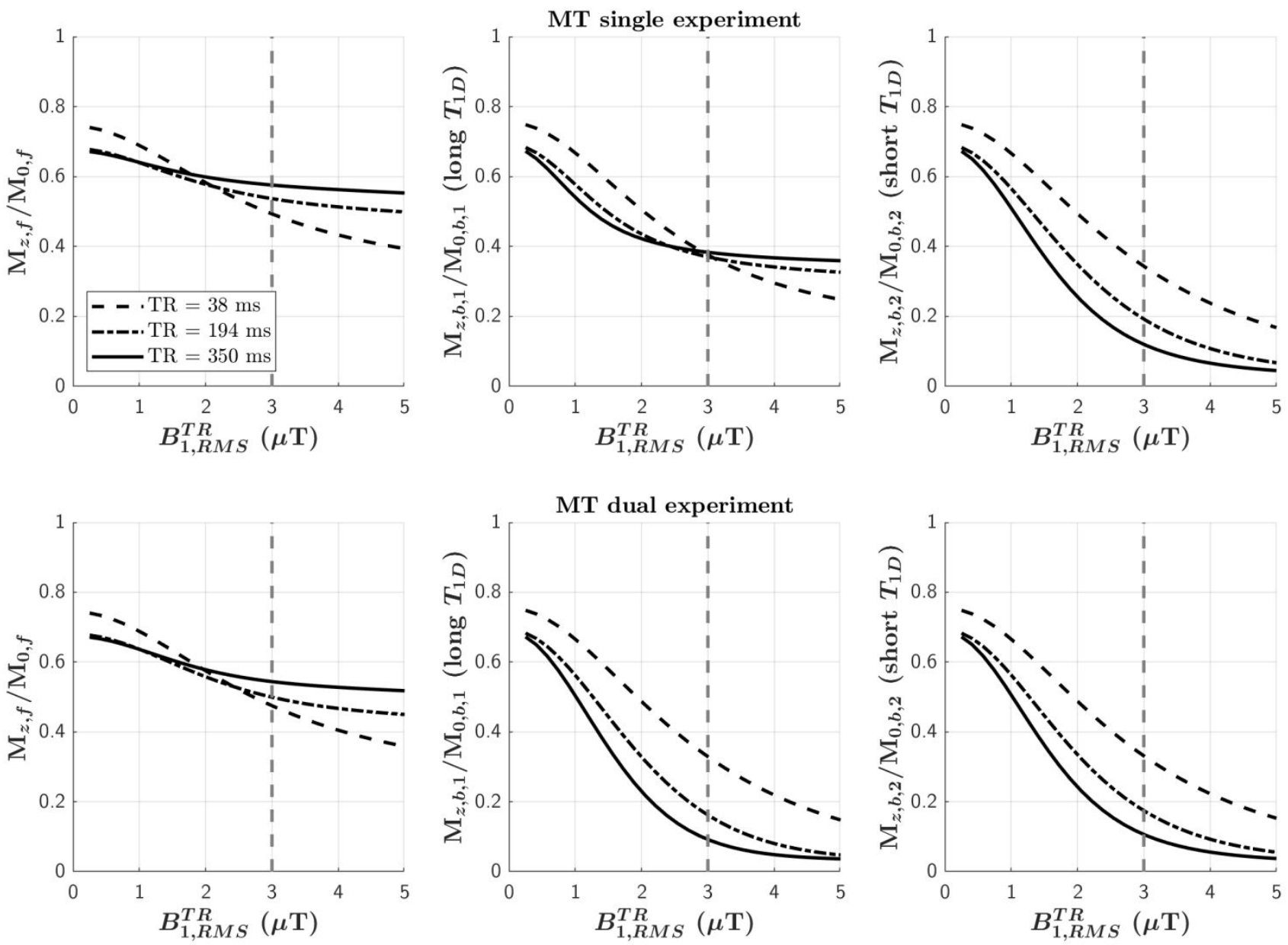

Figure S1: Simulations of normalized longitudinal magnetizations in steady-state of the free pool (left column) and bound pools (long $T_{1 \mathrm{D}}$ : middle column; short $\mathrm{T}_{1 \mathrm{D}}$ : right column) in the single MT (top row) and dual MT (bottom row) experiments as a function of $\mathrm{B}_{1, \mathrm{RMs}}{ }^{\mathrm{TR}}$ and for TR of 38,194 and $350 \mathrm{~ms}$. Dashed grey lines indicate the working nominal power as used in experiments $(3 \mu \mathrm{T})$. Simulated tissue parameters: $R_{1, f} / R_{1, b}=1.0 / 1.0 \mathrm{~s}^{-1}, T_{2, f}=22.0 \mathrm{~ms}, T_{2, b}=10.0 \mu \mathrm{s}, R$ $=19.0 \mathrm{~s}^{-1}, \mathrm{M}_{0, \mathrm{f}} / \mathrm{M}_{0, \mathrm{~b}}=1.0 / 0.16, \mathrm{~T}_{1 \mathrm{D}, 1} / \mathrm{T}_{1 \mathrm{D}, 2}=10.0 / 0.4 \mathrm{~ms}$ and $\mathrm{f}_{\mathrm{D}}=0.5$. 
Description of Figure S1: Following the Provotorov theory of RF absorption in solids, it is now well understood that the dipolar order acts against the saturation of the Zeeman order (the so-called magnetization of the macromolecular pool; i.e. $\mathrm{M}_{\mathrm{z}, \mathrm{b}}$ ), and that strong RF saturation performed at a single frequency offset promotes conversion of Zeeman order into dipolar order, hence hindering efficient saturation. Conversely when the RF saturation is performed with a dual-frequency offset (symmetric with respect to the center of the macromolecular lineshape), there is no creation of dipolar order, and the RF saturation is more efficient (although still limited by conventional $\mathrm{T}_{1}$ relaxation effects). Moreover, short $\mathrm{T}_{1 \mathrm{D}} \mathrm{S}$ make dipolar order vanishingly small for values below the millisecond range and RF saturation power achievable with typical clinical systems, hence promoting a long $T_{1 D}$ weighting in clinical ihMT imaging. These effects are altogether demonstrated in the following simulations of the steady state magnetization of the free water (left column) and the two macromolecular pools (middle column: long $T_{1 D}$; right column : short $T_{1 D}$ ) as a function of $B_{1, R M s}{ }^{T R}$ for both the single (upper row) and the dual RF saturation conditions (lower row).

Inspecting the curves from right to left: the Zeeman magnetization corresponding to the short $T_{1 D}$ component $\left(M_{z, b, 2}\right)$ shows a rather strong attenuation for high $\mathrm{B}_{1, \mathrm{RMS}}{ }^{\mathrm{TR}}$ values for both the single and dual RF saturation. This is expected for short $\mathrm{T}_{1 \mathrm{D}} \mathrm{S}$ for which a very low amount of dipolar order is generated. It appears from the three simulated curves that a concentrated RF energy deposition (solid line) attenuates more efficiently the bound pool magnetization than a rather distributed RF energy (dashed line), eventually leading to an almost complete saturation (ideally obtained for $M_{z, b}=0$ ) for $B_{1, R M s}{ }^{T R}>3-4 \mu T$. Obviously, the longitudinal relaxation prevents a perfect saturation of the bound pool, and it is expected that $\mathrm{M}_{z, \mathrm{~b}}$ stabilizes to a non-zero value for high $\mathrm{B}_{1, \mathrm{RMS}}{ }^{\mathrm{TR}}$, as observed.

The Zeeman magnetization corresponding to the long $T_{1 D}$ component $\left(\mathrm{M}_{z, \mathrm{~b}, 1}\right)$ show the same behavior for the dual frequency saturation: the magnetization is almost perfectly saturated for high $\mathrm{B}_{1, \mathrm{RMS}}{ }^{\mathrm{TR}}$ and concentrated energy deposition (solid line). Once again this is indeed expected because almost no dipolar order is generated in this case (no dipolar order would be created for a simultaneous dual frequency saturation, but a small amount is expected to remain for the frequency alternated scheme). However, for the single frequency saturation, $\mathrm{M}_{z, \mathrm{~b}, \mathrm{i}}$ is significantly higher (i.e. less attenuated) in comparison to the dual frequency case because its dipolar order acts against the saturation of the Zeeman order. Of interest, the case of high energy concentration (solid line) deviates the most from the single frequency case because the high RF irradiation power used during the MT pulses generates more dipolar order than the lower RF irradiation used in the distributed case, eventually leading to a crossing of the curves, and a "saturation" of the RF absorption of the macromolecular pool at high $\mathrm{B}_{1, \mathrm{RMS}}{ }^{\mathrm{TR}}$ for the concentrated energy deposition. Looking at these curves it appears clear that the dipolar order can be a much stronger opponent to the full saturation of the macromolecular magnetization than usual $\mathrm{T}_{1}$ relaxation effects.

Finally, the Zeeman magnetization of the liquid pool $\left(\mathrm{M}_{\mathrm{z}, \mathrm{f}}\right)$ reflects the mechanisms that we have just described at the macromolecular pool levels thanks to the magnetization transfer effects. The attenuation of the magnetization reaches a plateau (i.e. "saturates") only in the case of distributed energy deposition, and this is ultimately what is observed when looking at the ihMT signal (or ihMTR) in our experiments. 


\section{Offset frequency $(\Delta f)$}

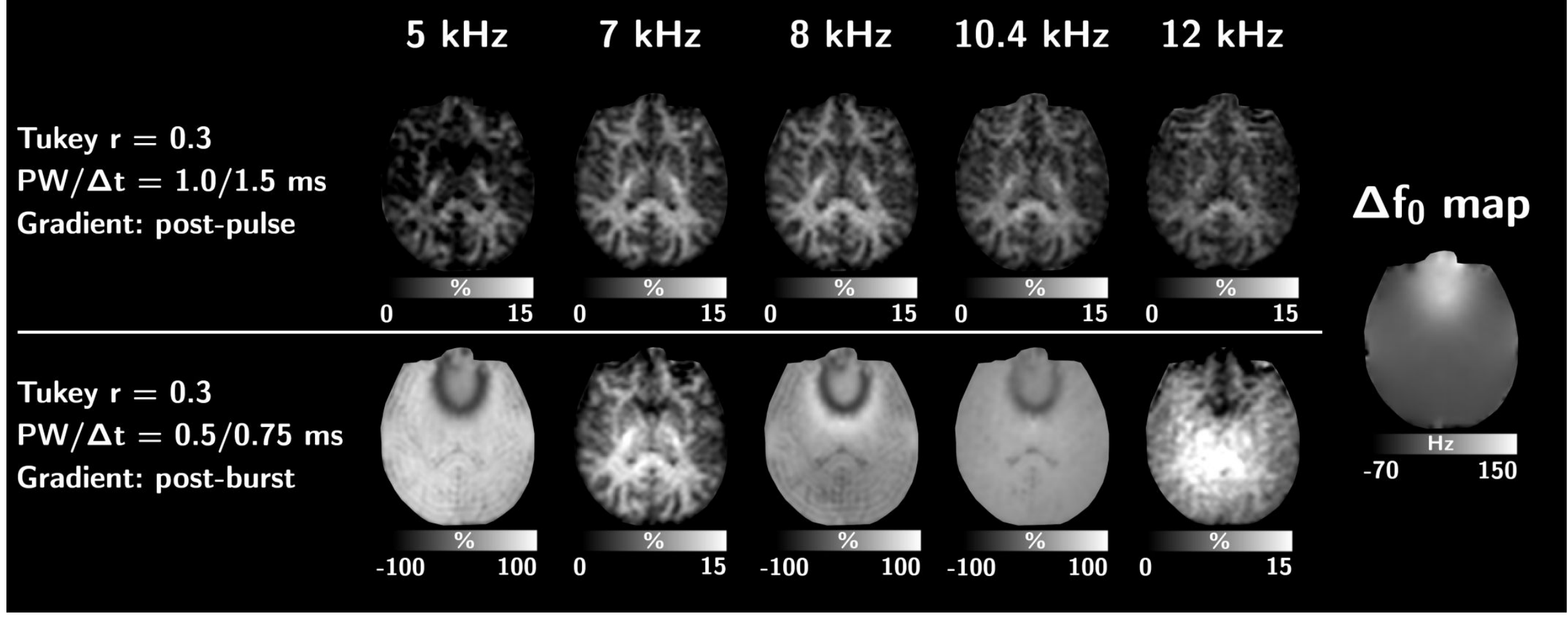

Figure S2: ihMTR maps as a function of the preparation pulses offset frequency, generated with $P W / \Delta t=1.0 / 1.5 \mathrm{~ms}\left(\mathrm{~N}_{\mathrm{p}}=8\right)$ with post-pulse spoiling gradients (top) and $\mathrm{PW} / \Delta \mathrm{t}=0.5 / 0.75 \mathrm{~ms}\left(\mathrm{~N}_{\mathrm{p}}=16\right)$ with a single post-burst spoiling gradient (bottom; note the changes in image dynamic), and corresponding $\Delta \mathrm{f}_{0}$ map. Common ihMT sequence parameters: $B_{1 \text {, peak }}=22.0 \mu \mathrm{T}, B_{1, R M s}{ }^{\top R}=3.0 \mu \mathrm{T}, \mathrm{TR}=350 \mathrm{~ms}$, $\mathrm{TR}_{\text {sub }}=6.5 \mathrm{~ms}$, number of $\mathrm{GRE}$ segments $=52$, readout flip angle $=5^{\circ}$. 


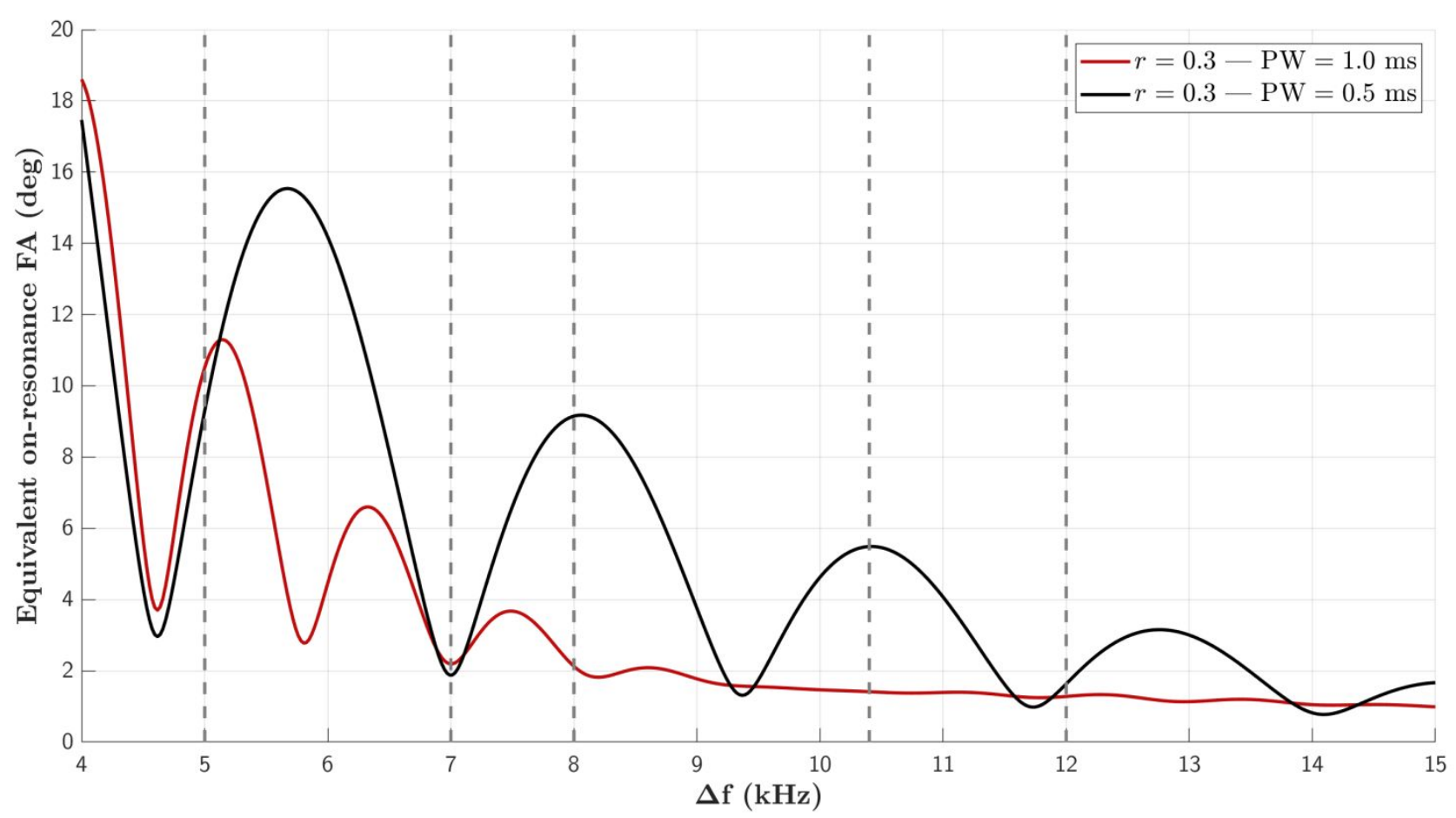

Figure S3: Equivalent on-resonance flip angle of a single off-resonance Tukey-shaped ( $\left.r=0.3, B_{1, \text { peak }}=22.0 \mu \mathrm{T}\right)$ pulse as a function of its frequency offset for $\mathrm{PW}=1.0 \mathrm{~ms}$ (red) and PW=0.5 ms (black). Curves were generated by simulations of the Bloch equations, and calculated from the longitudinal magnetization at the end of the pulse application as $\cos ^{-1}\left(M_{2} / M_{0}\right)$ (starting from an equilibrium state $M_{2}=M_{0}$, and neglecting relaxation and $M T$ effects). Dashed gray lines indicate experimental off-resonance frequencies (Fig. S2).

Descriptions of Figures S2 and S3: Figure S2 presents experimental ihMTR maps obtained with different preparation schemes $\left(P W / \Delta t=1.0 / 1.5 \mathrm{~ms}\left(\mathrm{~N}_{\mathrm{p}}=8\right)\right.$ with post-pulse spoiling gradients (top) and $P W / \Delta t=0.5 / 0.75 \mathrm{~ms}\left(N_{p}=16\right)$ with a single post-burst spoiling gradient) at different offset frequencies. Strong artifacts appear in the scheme without post-pulse spoiling gradient. The effects also correlate with the apparent on-resonance flip angle reflecting the direct saturation effects (calculated over a single off-resonance pulse; Figure S3, black curve). As such, it appears that the post-pulse spoiling gradient is mandatory to guarantee high image quality for short pulses (Figure S2; top row), although low frequency offsets may still yield a deteriorated image quality (Figure S2; top row for $\Delta f$ $=5 \mathrm{kHz}$ ) due to direct saturation effects (Figure S3, red curve). 